Prepared for the U. S. Department of Energy under Contract DE-AC05-76RL01830

\title{
Utilizing Electric Vehicles to Assist Integration of Large Penetrations of Distributed Photovoltaic Generation
}

FK Tuffner

FS Chassin
MCW Kintner-Meyer

K Gowri

November 2012

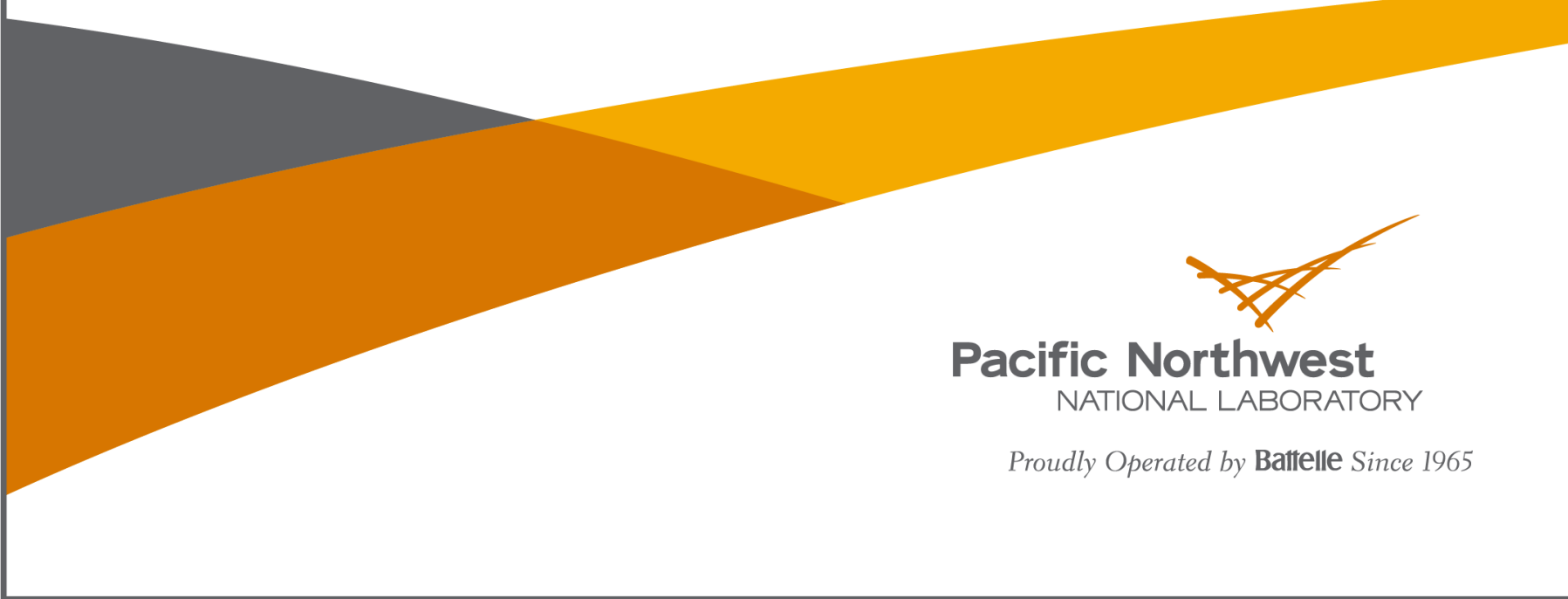




\title{
DISCLAIMER
}

This report was prepared as an account of work sponsored by an agency of the United States Government. Neither the United States Government nor any agency thereof, nor Battelle Memorial Institute, nor any of their employees, makes any warranty, express or implied, or assumes any legal liability or responsibility for the accuracy, completeness, or usefulness of any information, apparatus, product, or process disclosed, or represents that its use would not infringe privately owned rights. Reference herein to any specific commercial product, process, or service by trade name, trademark, manufacturer, or otherwise does not necessarily constitute or imply its endorsement, recommendation, or favoring by the United States Government or any agency thereof, or Battelle Memorial Institute. The views and opinions of authors expressed herein do not necessarily state or reflect those of the United States Government or any agency thereof.

\author{
PACIFIC NORTHWEST NATIONAL LABORATORY \\ operated by \\ BATTELLE \\ for the \\ UNITED STATES DEPARTMENT OF ENERGY \\ under Contract DE-AC05-76RL01830
}

Printed in the United States of America

Available to DOE and DOE contractors from the Office of Scientific and Technical Information,

P.O. Box 62, Oak Ridge, TN 37831-0062; ph: (865) 576-8401 fax: (865) 576-5728 email: reports@adonis.osti.gov

\begin{abstract}
Available to the public from the National Technical Information Service, U.S. Department of Commerce, 5285 Port Royal Rd., Springfield, VA 22161 ph: (800) 553-6847 fax: (703) 605-6900

email: orders@ntis.fedworld.gov online ordering: http://www.ntis.gov/ordering.htm
\end{abstract}

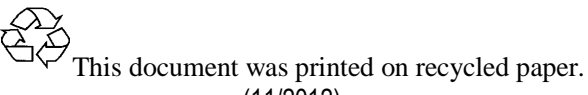

$(11 / 2012)$ 


\section{Utilizing Electric Vehicles to Assist Integration of Large Penetrations of Distributed Photovoltaic Generation}

FK Tuffner FS Chassin
MCW Kintner-Meyer

K Gowri

November 2012

Prepared for

the U. S. Department of Energy

under Contract DE-AC05-76RL01830

Pacific Northwest National Laboratory

Richland, Washington 99352 


\section{Executive Summary}

\section{Introduction and Motivation}

This analysis provides detailed distribution-level insights into the leveraging potential of distributed rooftop photovoltaic (PV) technologies and electric vehicle (EV) charging. Either of the two technologies by themselves - at some high penetrations - may cause some voltage control challenges or overloading problems, respectively. But when combined, there could be synergistic effects, at least intuitively, whereby one technology mitigates the negative impacts of the other. High penetration of EV charging may overload existing distribution system components, most prominently the secondary transformer. If PV technology is installed at residential premises or anywhere downstream of the secondary transformer, it will provide another electricity source, thus relieving the loading on the transformers. Another synergetic or mitigating effect could be envisioned when high PV penetration reverses the power flow upward in the distribution system (from the homes upstream into the distribution system). Protection schemes may then no longer function as designed and voltage violations (exceeding the voltage upper limit of the American National Standards Institute (ANSI) voltage range) may occur. In this particular situation, EV charging could consume the generated energy from the PV, such that the reversal of power flow can be reduced or alleviated. Given these potential mutual synergistic behaviors of PV and EV technologies, this project attempted to quantify the benefits of combining the two technologies.

Furthermore, of interest was how advanced EV control strategies may influence the outcome of the synergy between EV charging and distributed PV installations. Particularly, California utility companies with high penetration of distributed PV technology, who have experienced voltage control problems, are interested in how intelligent EV charging could support or affect the voltage control challenges.

\section{Methodology and Scope}

The analysis explored a small parameter space of different penetration levels of electric vehicles and distributed solar generation and the impacts these penetrations have on a distribution system. The investigation was performed by means of a power flow simulation of an Institute of Electrical and Electronics Engineers (IEEE) test feeder. To keep this study generic, a readily available generic IEEE feeder was utilized, rather than a specific feeder of a specific distribution company. The IEEE 123-node radial distribution feeder was used in this study [IEEE 1991]. This feeder represents a small distribution feeder with several regulators to maintain system operating voltage. Figure ES.1 shows the representative one-line diagram for this distribution feeder. 


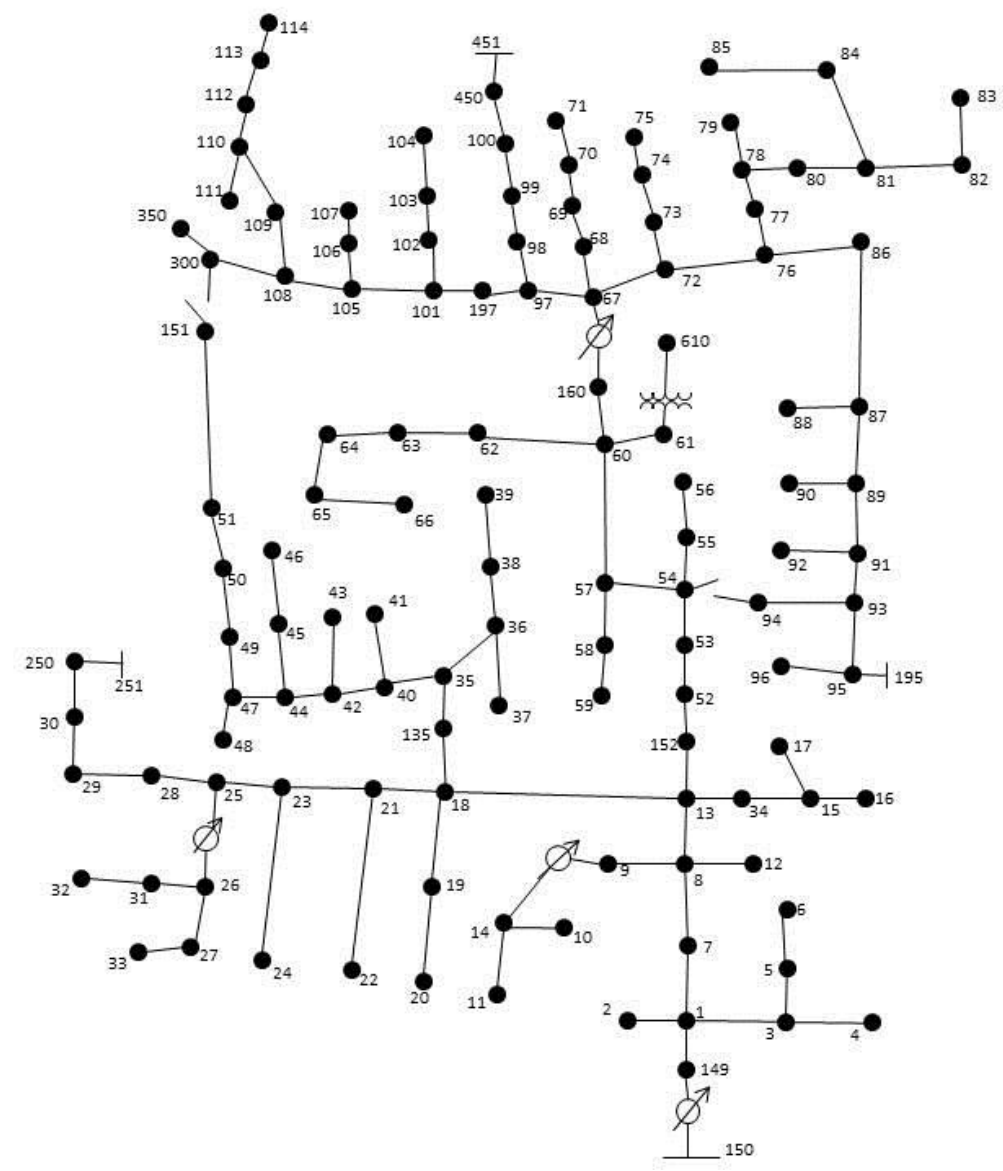

Figure ES.1. IEEE 123-node Feeder, One-line Diagram

The IEEE 123-node feeder is defined for a constant load. The original load conditions were replaced with explicit thermal and load representations of residences and commercial buildings, and EV charging behavior that is defined by EV owners' driving patterns. The feeder was populated with 1251 residential homes and a few light commercial buildings. Building characteristics and weather data for the Phoenix, Arizona region were used to create a feeder representative of high solar electricity production region.

The power flow simulations with the specific EV charging strategies were implemented in the GridLAB$\mathrm{D}^{\mathrm{TM}}$ distribution software [GridLAB-D 2012]. GridLAB-D performs time-series simulations of various aspects of the distribution power system. With time varying weather information, customer usage information, and bulk-transmission information, impacts on the power system can be examined for multiple periods of the day. Impacts of increased demand in the late afternoon and evening as a consequence of increasing market adoption of electric vehicles and the mitigating influence of PV generation can be readily modeled.

The key outcomes of this analysis can be summarized as follows:

- EV and PV can operate synergistically in a distribution system. EV charging can provide additional load under high voltage conditions to reduce the excess voltage. PV resources provide an additional power injection, mitigating overload conditions on secondary transformers under high EV assumptions 
- The leveraging sensitivity was analyzed to be low, meaning that it would take significant amounts of the mitigating technology penetration to achieve an appreciable effect

- The EV charging strategies showed mixed results. The frequency-based EV charging strategy revealed the decoupled effect between the distribution system dynamics and that of the bulkpower system. The voltage-based control strategy showed improved results, because it was sensitive to changing local voltage conditions. Vehicle-to-Grid (V2G) could be detrimental to high voltage conditions in distribution system feeders because it turns an electric vehicle into a generator at times. EV smart charging (frequency or voltage-based) showed only minor benefits in mitigating this impact

- The caveat of this analysis was that these results are only applicable for the 123-node IEEE feeder with some assumptions. It is difficult to extrapolate these insights to other distribution feeders in a particular service territory, as many of the assumed conditions, such as distribution system topology, customer composition, and overall component sizing guidelines vary over a wide range

- Furthermore, another caveat is that some of the PV inverter technology may in the future be equipped with a four quadrant Volt/Var control, which may provide sufficient voltage controllability in the PV inverter. If each PV array or each PV installation could be equipped with such a smart inverter, voltage issues in distribution system may largely be solved.

\section{Caveats}

This is one of the initial detailed distribution-level studies that explore the synergies between distributed $\mathrm{PV}$ and EV charging. To set the right expectation for the outcome of this study, the authors would like to point out that the parameter space for this analysis is very large, requiring literally thousands of the scenarios of different distribution system topologies and loadings on individual feeders and segments of the distribution system. Just the vastness in diversity of distribution system topology and customer composition in the system, plus the diversity in distribution system design guidelines that recommend sizing of distribution components spans a very large parameter space, which this study cannot address in its entirety. Only a small piece of this much larger analysis 'picture' can be addressed in this report due to limited funding resources. We chose one and only one distribution system (IEEE 123-node system) and made assumptions regarding the existing loading for the end-user nodes. Furthermore, we selected a U.S. southwestern location (Phoenix, AZ) to maximize the solar electricity output by the distributed PV arrays. Overall within reasonable selection ranges, we created a scenario that was certainly favorable to high PV electricity output and high overall loading in the entire distribution feeders. Because of the targeted scope of this analysis (one feeder design, one location), the authors caution against extrapolating the results outside the context of the scenario definitions and underlying assumptions. The basic models and use of the GridLAB-D software package allow further scenarios and assumptions to be explored. The results should be considered as favorable for PV impact because of the high solar insolation in the Southwestern region. Therefore, the impacts of PV may be considered optimistic compared to regions with less solar insolation, such as the Midwestern and East Coast regions. However, the used 123-node feeder is relatively "stiff" with relatively short distances and low impedance between nodes. Distribution systems with long feeders may show a higher sensitivity with respect to voltage deviations and therefore the synergy between PV generation and EV charging may be more pronounced. 


\section{Acknowledgement}

The authors wish to thank Dr. Marcelo Elizondo, Dr. Chunlian Jin, and Mr. Jason Fuller at the Pacific Northwest National Laboratory for their assistance in this project. They were helpful in generating the balancing data used as a basis for the study, as well as the modeling script used to simulate the various systems.

The authors would also like to thank Mr. Dan Ton of the U.S. Department of Energy, Office of Electricity Delivery and Energy Reliability, and Mr. Lee Slezak with the U. S. Department of Energy, Office of Energy Efficiency and Renewable Energy, Vehicle Technologies Programs, for funding this research project. 


\section{Contents}

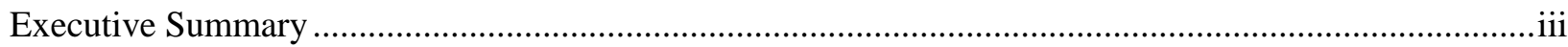

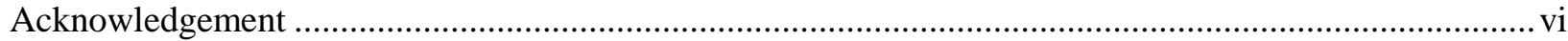

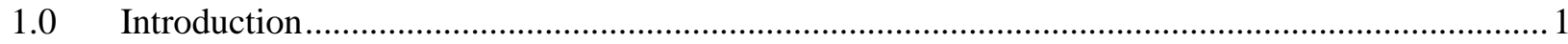

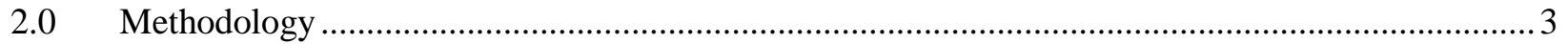

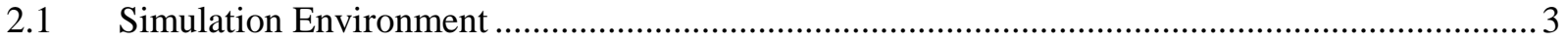

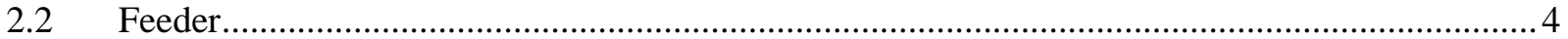

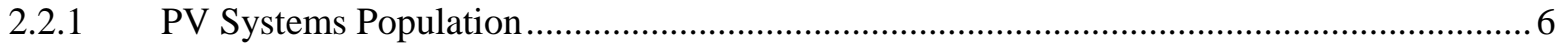

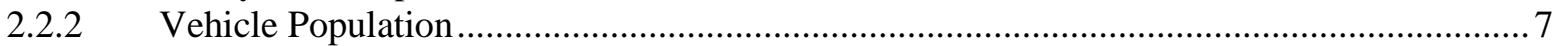

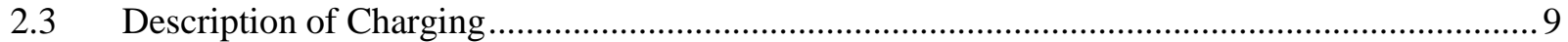

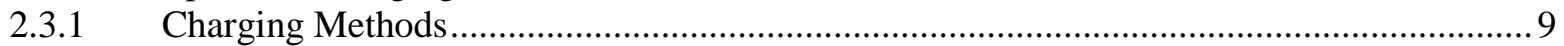

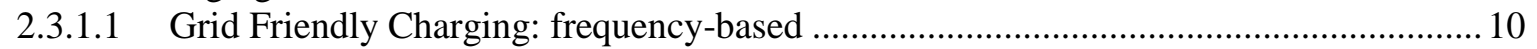

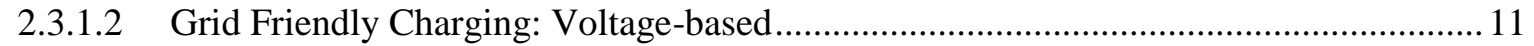

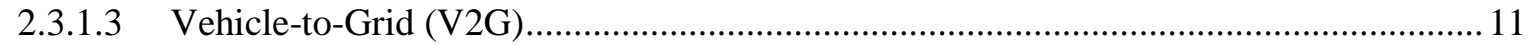

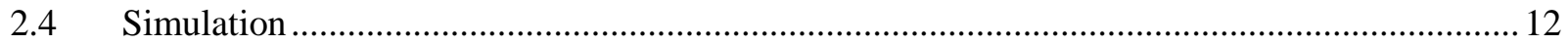

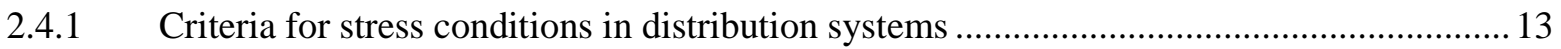

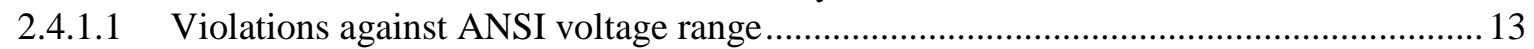

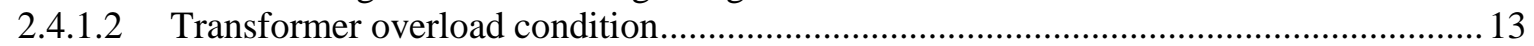

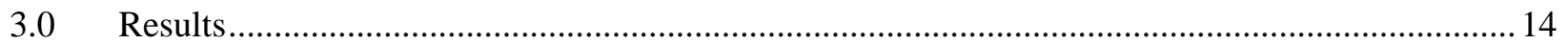

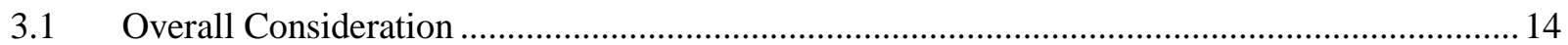

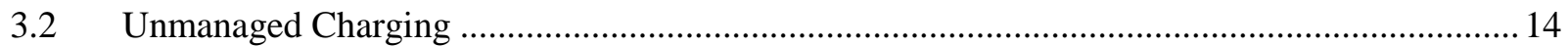

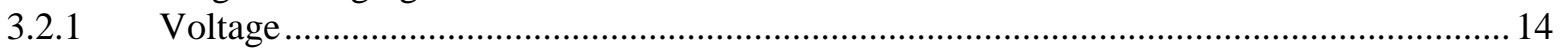

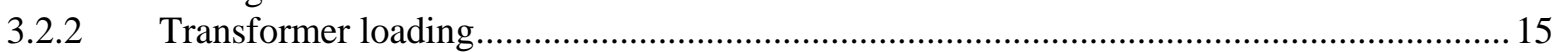

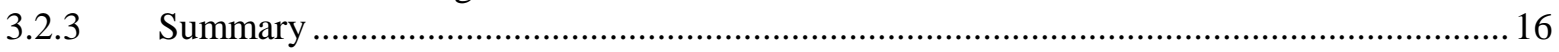

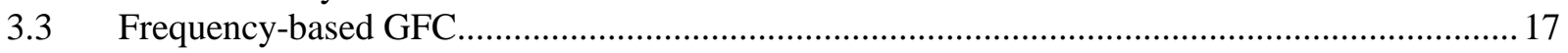

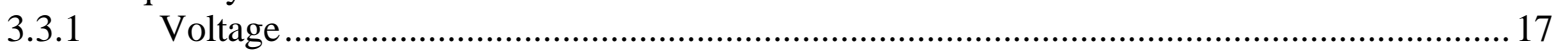

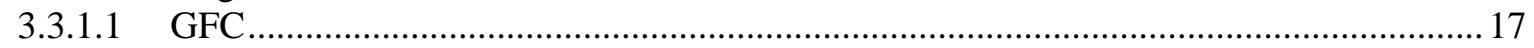

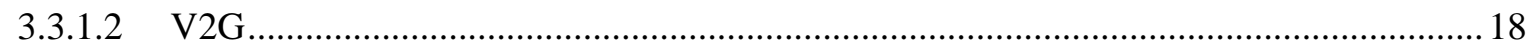

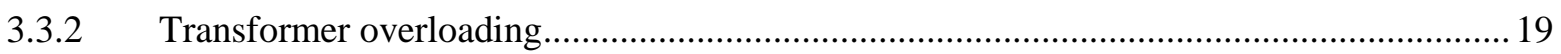

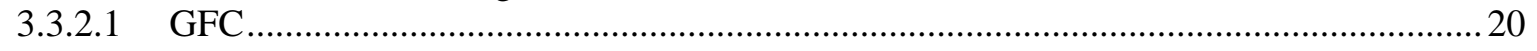

3.3.2.2 V2G

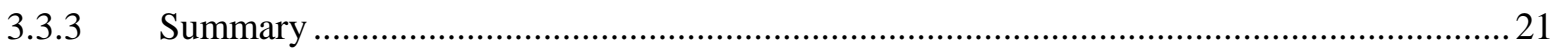

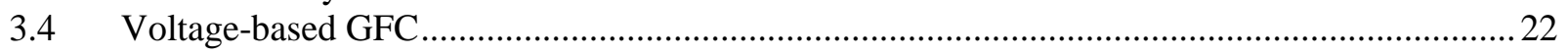

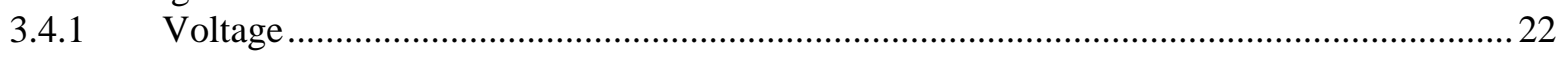

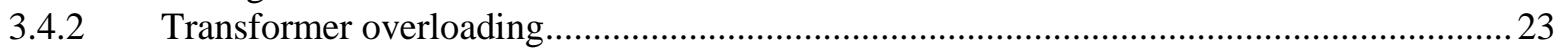

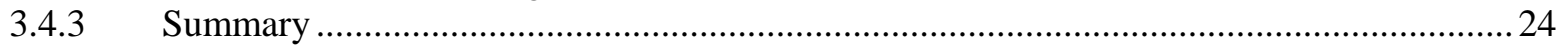

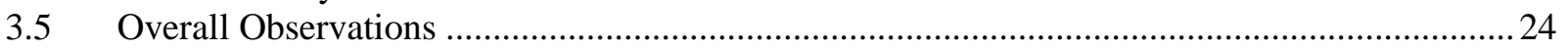

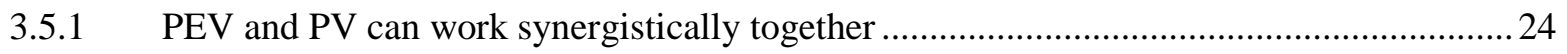

3.5.2 Impact of charging control strategies and $\mathrm{V} 2 \mathrm{G}$ applications ........................................25

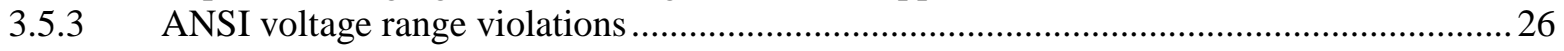

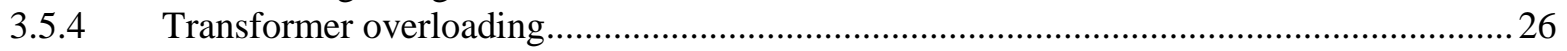

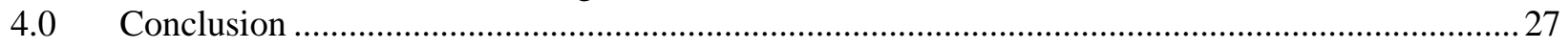

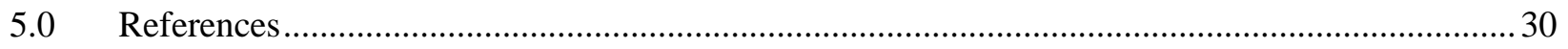




\section{Figures}

Figure ES.1. IEEE 123-node Feeder, One-line Diagram ...................................................................

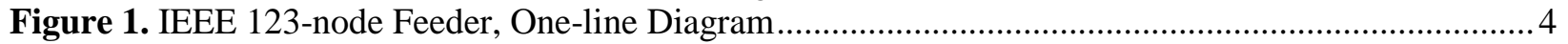

Figure 2. Voltage violations for measured nodes on the 123-node system.......................................... 6

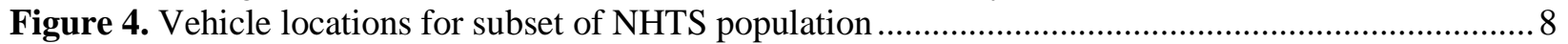

Figure 5. Number of vehicles charging during a typical simulation day .............................................. 9

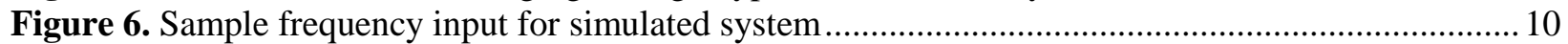

Figure 7. Example charger power for GFC (top) and V2G (bottom) ................................................... 12

Figure 8. Time outside ANSI limits for unmanaged charging and increased PV on node 66 ................. 15

Figure 9. Hours transformer is overloaded for unmanaged charging and increased PV ........................... 16

Figure 10. Time outside ANSI limits for GFC frequency-based charging and increased PV ................. 18

Figure 11. Time outside ANSI limits for V2G frequency-based charging and increased PV ................. 19

Figure 12. Hours transformer is overloaded for GFC frequency-based charging and increased PV .........20

Figure 13. Hours transformer is overloaded for V2G frequency-based charging and increased PV .........21

Figure 14. Time outside ANSI limits for V2G voltage-based charging and increased PV....................22

Figure 14. Hours transformer is overloaded for V2G voltage-based charging and increased PV ............23

Figure 15. Maximum PEV population for given solar population and no additional transformer

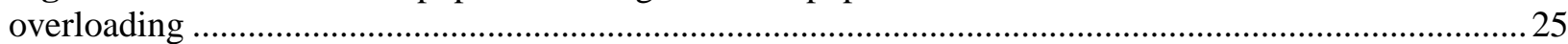

\section{Tables}

Table 1. Photovoltaic properties for different solar penetration levels .................................................. 7

Table 2. Number of electric vehicles deployed per penetration level ................................................... 7 


\subsection{Introduction}

Renewable generation sources are becoming a significant aspect of the modern power system. Domestic energy security, as well as environmental effects, is increasing the deployment of renewable generation sources. A total of 38 states have legislation or goals in place to have a significant portion of their energy come from renewable or alternative energy sources (renewable portfolio standards) [C2ES, 2012]. With such wide-spread proliferation of renewable generation sources, their impacts and benefits to the power system are becoming more apparent.

Photovoltaic (PV) solar generation systems are one renewable energy source gaining a lot of popularity. Their decreased cost and less stringent installation requirements make the deployment in residential neighborhoods very appealing. Through net-metering practices and other utility-based incentives, more and more rooftop PV arrays are being deployed. Their impact on the power system, especially the distribution-level power system, is becoming of greater interest.

Plug-in hybrid electric (PHEV) and plug-in electric vehicles (PEV) are also becoming more prevalent on the system. As technology matures and customer acceptance increases, the impacts and benefits of electric vehicles are also of concern. Electric vehicles have the potential to be a significant new load on the system that may burden the power system, both at the transmission and distribution level. However, they also represent a significant capacity of distributed energy storage that can provide services to the grid, even if they are only charged.

Electric vehicles and renewable generation resources could potentially be synergetic to each other, offsetting some of the impacts to the electricity infrastructure. One recent study detailed how the variability in wind generation can increase the balancing requirements on the bulk power system [Kintner-Meyer et al. 2010, Makarov et al. 2010]. Subsequent work investigated using electric vehicles to help meet and mitigate these additional balancing requirements [Tuffner and Kintner-Meyer, 2011]. By utilizing a variable charge rate and grid sensing technology, a fraction of the light duty vehicle fleet in the Pacific Northwest United States could meet the additional balancing requirements and offset the variability associated with a large deployment of wind. Rather than both additions (additional wind and increased PEVs) causing detrimental effects on the system, the two technologies are synergized to provide benefits to the power system.

Large penetration of rooftop PV generation has its own set of challenges. The variability in the output is of significant concern, as is the overall impact on the distribution power system through powerflow and equipment impacts. With significant deployments of photovoltaics, the local power system can often experience voltage rise and unexpected reverse power flow conditions. These reverse power flow conditions have the potential to even exceed the limit rating of transformers, thereby decreasing their lifespan.

Electric vehicles often produce an opposite effect. The increased load depresses voltage in areas of the distribution grid. The increased demand also has a possibility to overload distribution transformers, especially in older neighborhoods [Kintner-Meyer et al. 2007]. Coincident demand, typically associated with all of the electric vehicles arriving home and starting to charge at nearly the same time, can exacerbate this problem. 
The problems posed by the distributed PV and increased PEVs appear complimentary. PV is providing power to the local system and increasing voltage. PEVs are increasing local loads and decreasing voltage. With the proper combination of these two technologies, a "power system neutrality" may exist. This report will investigate the impacts of increased PV generation, increased PEV load, and their combination on a distribution power system. The insights of this study should aid the integration of the two technologies to provide the greatest benefit to the power grid, while promoting the goals of energy security and decreased environmental impacts. This insight may be of particular interest to distributionlevel utilities either looking to incentivize large deployments of PV and PEV, or who operate in regions of high penetration PEV and PV. This report investigates a few scenarios of the PV/PEV integration scenario, but provides a framework for further study. The particular techniques applied could be utilized on specific systems to extend the generic results of this report to a particular utility's concerns. 


\subsection{Methodology}

To investigate the potential benefits electric vehicles may provide to the integration of renewable generation, a typical feeder with these new technologies must be simulated. For maximum benefit to an individual utility, such simulations would require the topology and load information for a specific feeder on their distribution system. However, to keep this study generic, a readily available, generic Institute of Electrical and Electronics Engineers (IEEE) radial test feeder was utilized. Different penetration levels of electric vehicles and distributed solar generation were simulated and the impacts these technologies had on the distribution system were examined.

\subsection{Simulation Environment}

Simulations for the interactions and impacts of the photovoltaic and electric vehicle populations were conducted inside the GridLAB-D distribution software [GridLAB-D 2012]. GridLAB-D was developed at the Pacific Northwest National Laboratory for the Department of Energy to study new grid technologies on the distribution system. Using explicit models of different levels of the distribution power system, GridLAB-D has the capability to simulate the system all the way from the connecting substation to the final end-use load.

GridLAB-D performs time-series simulations of various aspects of the distribution power system. With time varying weather information, customer usage information, and bulk-transmission information, impacts on the power system can be examined for multiple periods of the day. Impacts of increased demand in the late afternoon and evening as a consequence of increasing market adoption of electric vehicles and the mitigating influence of PV generation can be studied.

One of GridLAB-D's specific capabilities for distribution power system analysis is the inclusion of a three-phase, unbalanced powerflow solver, especially in conjunction with the time-series simulations. Traditional power simulation programs were designed for transmission networks, where many assumptions and common practices simplify the equivalent system to a single wire. However, the differing load composition and nature of distribution networks requires all three phases of a normal power system to be explicitly simulated, especially as these loads vary throughout the day. This is especially true for residential deployments of both PV and PEV technologies. The technologies may not be deployed evenly on all three phases and variability in usage, especially in regards the PEV drive cycles, can create varying unbalanced conditions throughout the day. GridLAB-D simulates the interactions and imbalances between all three phases of the distribution network explicitly. This allows issues, such as unbalanced loading and increased losses, to be explicitly examined for systems of interest.

Another GridLAB-D capability that is useful for distribution-level studies is the detailed load and thermal models for commercial and residential buildings. Internal building temperatures are modeled to take into consideration thermal mass, solar gains, electric heat losses, and heating, ventilation and air-conditioning (HVAC) impacts. These explicit models provide a very detailed representation of distribution-level loads and their secondary impacts on the power system.

Weather information for the buildings and photovoltaic arrays is incorporated into GridLAB-D. Weather information is extracted from typical meteorological year (TMY) data put together by the National Renewable Energy Laboratory [NREL 2012]. Coupled with higher time-resolved solar information from 
the National Oceanic and Atmospheric Administration, this information provides a basis for the thermal and solar models in the GridLAB-D environment[NOAA 2012]. Different temperature and solar conditions affecting the PV generation of each of the distributed systems are modeled sub-hourly to estimate the overall impacts to the entire distribution system.

\subsection{Feeder}

The basis for all of the simulations is the underlying distribution power system, which was parameterized to represent a set of PV technology penetration and PEV market adoption.. A generic 123-node, radial distribution feeder established by IEEE was used in this study [IEEE 1991]. This feeder represents a small distribution feeder with several regulators to maintain system operating voltage. Figure 1 shows the representative one-line diagram for this distribution feeder.

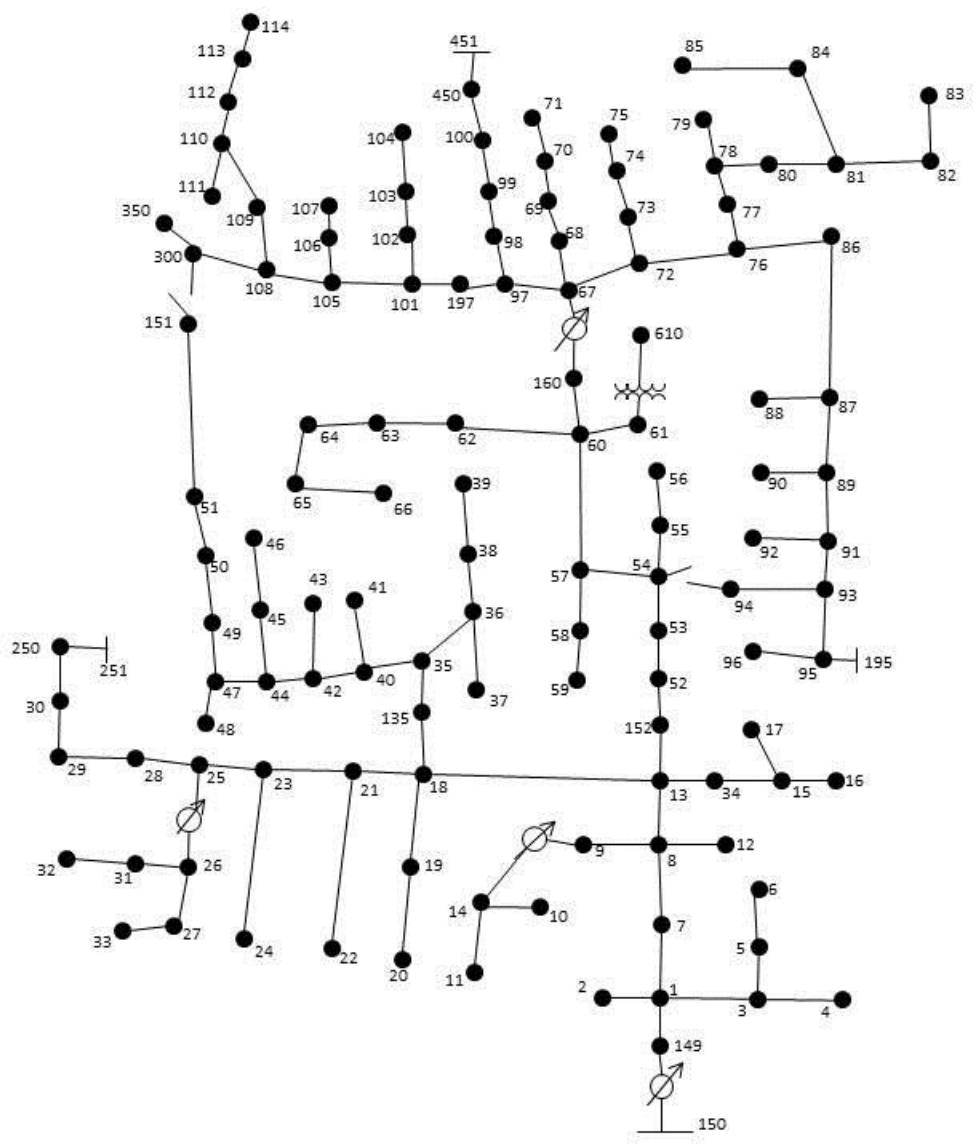

Figure 1. IEEE 123-node Feeder, One-line Diagram

By default, the IEEE 123-node feeder is defined for a constant load representing a standard peak load allocation study. To adapt the original load conditions for the purpose of this study, these loads needed to be replaced with explicit thermal and load representation of residences and commercial buildings. Using techniques developed in [Singh and Vyakaranam 2012], the feeder was then populated with 1251 residential homes and a few light commercial buildings. Building characteristics and weather data for the Phoenix, Arizona region were used to create a feeder representative of high solar electricity production region. 
In its initial state, the IEEE 123-node system was not fully suited for the combined PV/PEV study. The following minor changes were made to this feeder: 1) The overall peak feeder loading was increased slightly to 7.0 MVA to represent a feeder close to its components rate limits; and 2) A set of capacitors were added and voltage regulator settings changed, so that voltage levels for the base case with no electric vehicles or photovoltaics, would be close to the limits of the acceptable American National Standards Institute (ANSI) range: near $126 \mathrm{~V}$ at the start of the feeder and near $114 \mathrm{~V}$ at the end of the lines. With these adjustments, several nodes in the system would briefly drop below 107V (just under the limit of the emergency voltage range) a few minutes during the week-long simulations. These nodes represent an extremely sensitive location on the modified feeder and provided a location to evaluate the impacts of the PV and PEV deployments.

Figure 2 shows an indication of the amount of time various points on the 123-node system were outside the acceptable "Range A" voltage conditions of ANSI standard C84.1 [ANSI C84.1-2006]. The results of Figure 2 are shown in the product term of "volt-minutes", which is representative of a node being 1-volt out of range for 1-minute. As an example, an excursion of 2.0 volts for one minute would count as 2.0 Volt-minutes of violation. This particular metric is explained later in the report. The figure shows the results of simulations with every house having a photovoltaic array. In the two cases of Figure 2, one population had no electric vehicles, while in the other, $40 \%$ of the population had an electric vehicle. The numbers on the $x$-axis correspond to the bus numbers of Figure 1 with "SPh" designation a single-phase electrical node, and " $3 \mathrm{Ph}$ " representing a three-phase electrical node. As the figure shows, there were a significant number of voltage violations on the system. To summarize the results for this study, a single node was selected as the average representation of the system. Node 66 was selected for all subsequent plots, and as such, will be a proxy for a set of voltage sensitive nodes. 


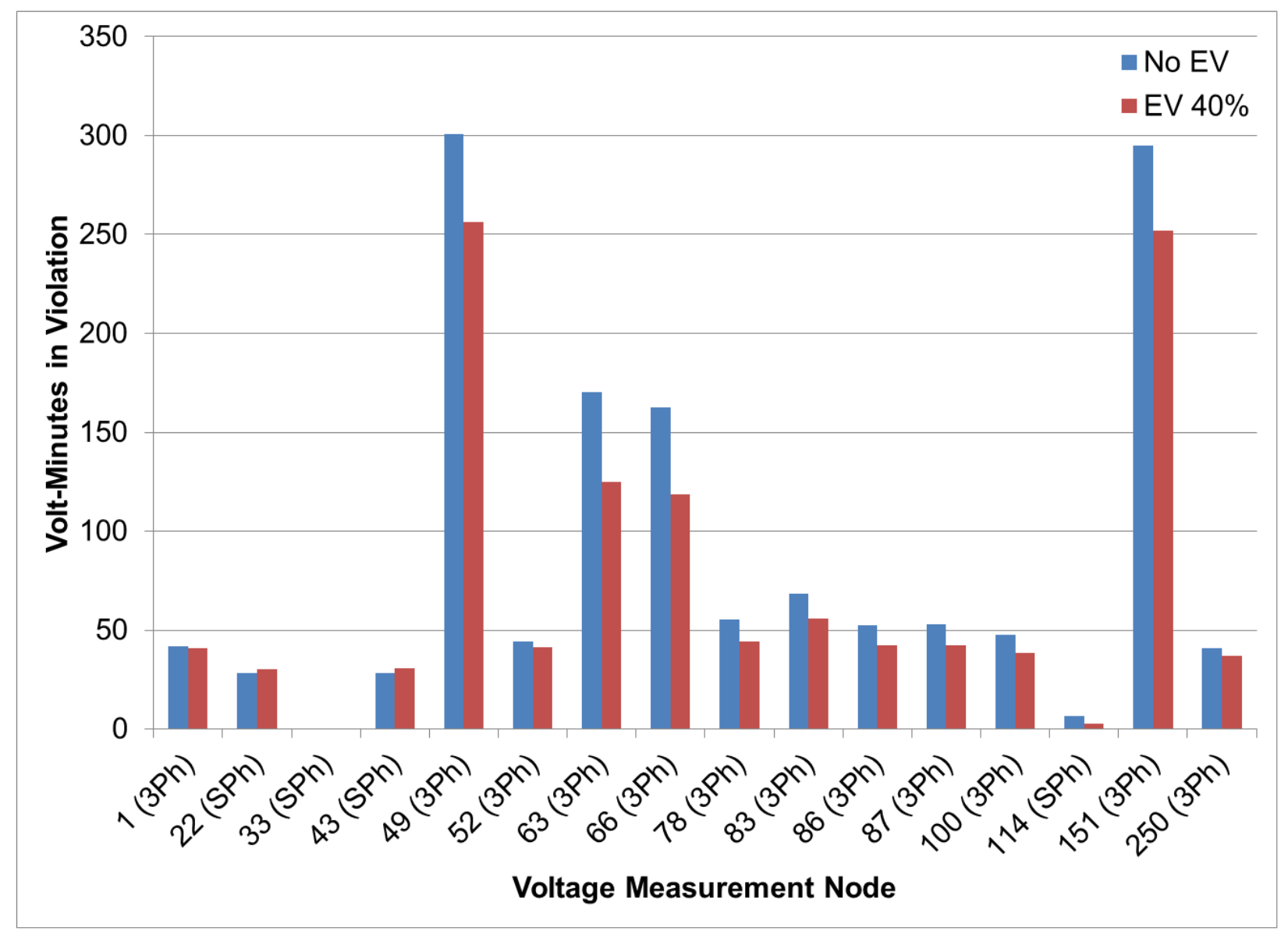

Figure 2. Voltage violations for measured nodes on the 123-node system

\subsubsection{PV Systems Population}

With the residential and commercial building basis complete, the feeder also needed to be populated with distributed solar generation. Varying levels of rooftop PV generation were placed on the buildings of the feeder. Penetration levels varied from no PV capacity to full deployment (meaning each residential and commercial customer have some PV capacity). Overall, this resulted in a little over $8 \mathrm{MW}$ of generating capacity. The PV models are simplified representations of single-crystal silicon photovoltaic arrays with an overall efficiency of $18 \%$, including the inverter losses. Ideal solar power-point tracking is assumed to convert received solar insolation data to generated power.

Each PV unit deployed was proportional to the floor area of the attached building, assumed to be directly proportional to the roof size of the building. Table 1 shows: the number of buildings with photovoltaic arrays; the size of an equivalent, single array; and the theoretical maximum power output of the equivalent array. An upper limit of $25 \mathrm{~m}^{2}\left(270 \mathrm{ft}^{2}\right)$ was selected for the arrays to ensure they were not too large. Given the residential and light commercial composition of the selected feeder, this array restriction is not unreasonable. PV arrays were connected directly to the utility grid, rather than through the local panel of the building. This deployment represents a common, sub-metered grid-tied integration of photovoltaic generation as used by many utilities offering special incentives for distributed generation owners. 
Table 1. Photovoltaic properties for different solar penetration levels

\begin{tabular}{|r|c|c|c|c|c|c|}
\cline { 2 - 7 } \multicolumn{1}{c|}{} & \multicolumn{7}{c|}{ Solar Penetration } \\
\cline { 2 - 7 } \multicolumn{1}{c|}{} & $0 \%$ & $20 \%$ & $40 \%$ & $60 \%$ & $80 \%$ & $100 \%$ \\
\hline Number of Houses & 0 & 324 & 687 & 1093 & 1251 & 1251 \\
Commercial Buildings & 0 & 13 & 24 & 30 & 36 & 43 \\
Array Size (ft $\left.{ }^{2}\right)$ & 0 & 111900 & 234400 & 337800 & 398000 & 435600 \\
Array Output (kW) & 0 & 2080 & 4360 & 6280 & 7390 & 8090 \\
\hline
\end{tabular}

\subsubsection{Vehicle Population}

The feeder was also populated with varying numbers of PEVs. Electric vehicles were represented by a fairly simple model. Vehicles simulated were assumed to be battery electric vehicles, similar to the Nissan Leaf, with an electric range of 110 miles. For the assumptions of the simulation, this equates to a 28.6-kWh battery. All charging scenarios were assumed to have an overall $90 \%$ charging efficiency. In scenarios of PEV discharge, the inverter was also assumed to have $90 \%$ efficiency (for a round-trip efficiency of $81 \%$ ). Simulations explicitly tracked the vehicle state of charge, so once a vehicle reached full capacity, it no longer would load the power system.

As with the solar deployments, the number of electric vehicles was varied from no vehicles to one vehicle per residential home (1251 total). Table 2 shows the specific vehicle counts for each electric vehicle penetration level. The vehicles were simulated under the assumption the car was the residence's primary form of transportation, so it was used every day for the normal commute. The vehicles were also assumed to only charge at home, as opposed to charging at work. All vehicles were assumed to be identical in capacity and efficiency, with only differences in their usage and charging strategy utilized.

Table 2. Number of electric vehicles deployed per penetration level

\begin{tabular}{|c|c|c|c|c|c|c|}
\cline { 2 - 7 } \multicolumn{1}{c|}{} & \multicolumn{6}{|c|}{ Electric Vehicle Penetration } \\
\cline { 2 - 7 } & $0 \%$ & $20 \%$ & $40 \%$ & $60 \%$ & $80 \%$ & $100 \%$ \\
\hline No. of Houses with EVs & 0 & 233 & 475 & 733 & 996 & 1251 \\
\hline
\end{tabular}

Individual vehicle behavior was governed by information extracted from the 2001 National Household Travel Survey (NHTS) [USDOT 2003]. Driving and availability schedules were selected so vehicles represented a variety of work schedules (day, night, and swing shifts). Because all vehicles were assumed to only have home charging available, trip distances were selected such that the 110-mile range of the PEV was sufficient. Figure 3 shows a small subset of the vehicle driving behavior. 


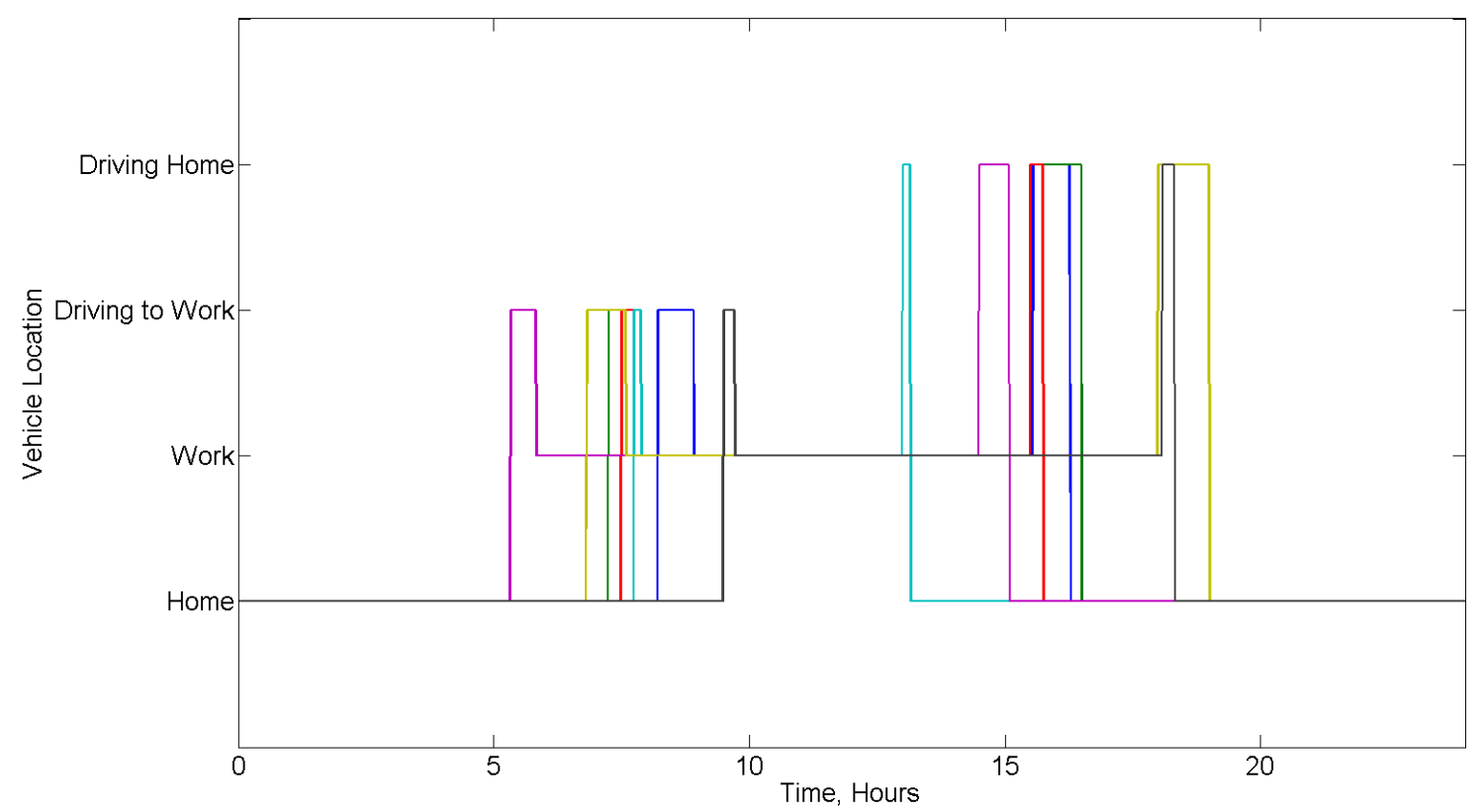

Figure 3. Vehicle locations for subset of NHTS population

Combining the NHTS data and the underlying assumptions of the different vehicles, Figure 4 is produced. Figure 4 shows the number of vehicles charging during a typical day of the simulation. This is different from what is usually shown for vehicle availability. In this study, charging at work was not considered. To increase daytime availability of electric vehicles, work schedules were adjusted to include swing (shift starts around 2 PM) and night shift workers (shift starts around 10 PM, often longer than 8 hours). The extra availability enables electric vehicles to help mitigate some of the feeder impacts observed during peak solar output. The four smaller peaks between $1 \mathrm{AM}$ and $12 \mathrm{PM}$ can be attributed to swing and night shift workers arriving home. Vehicles are charged using an "unmanaged charging" scheme, which will be described in the next section. This basically amounts to a constant power draw to charge the battery as soon as the vehicle is connected. Even with the attempts to diversify the work schedules, the largest group of vehicles is charging in the $6 \mathrm{PM}$ to midnight time interval. This is indicative that most of the vehicles are arriving home at that time and immediately starting to charge (based on the assumptions of the "unmanaged charging" method). Similar availability was applied to the other charging methods of this report. 


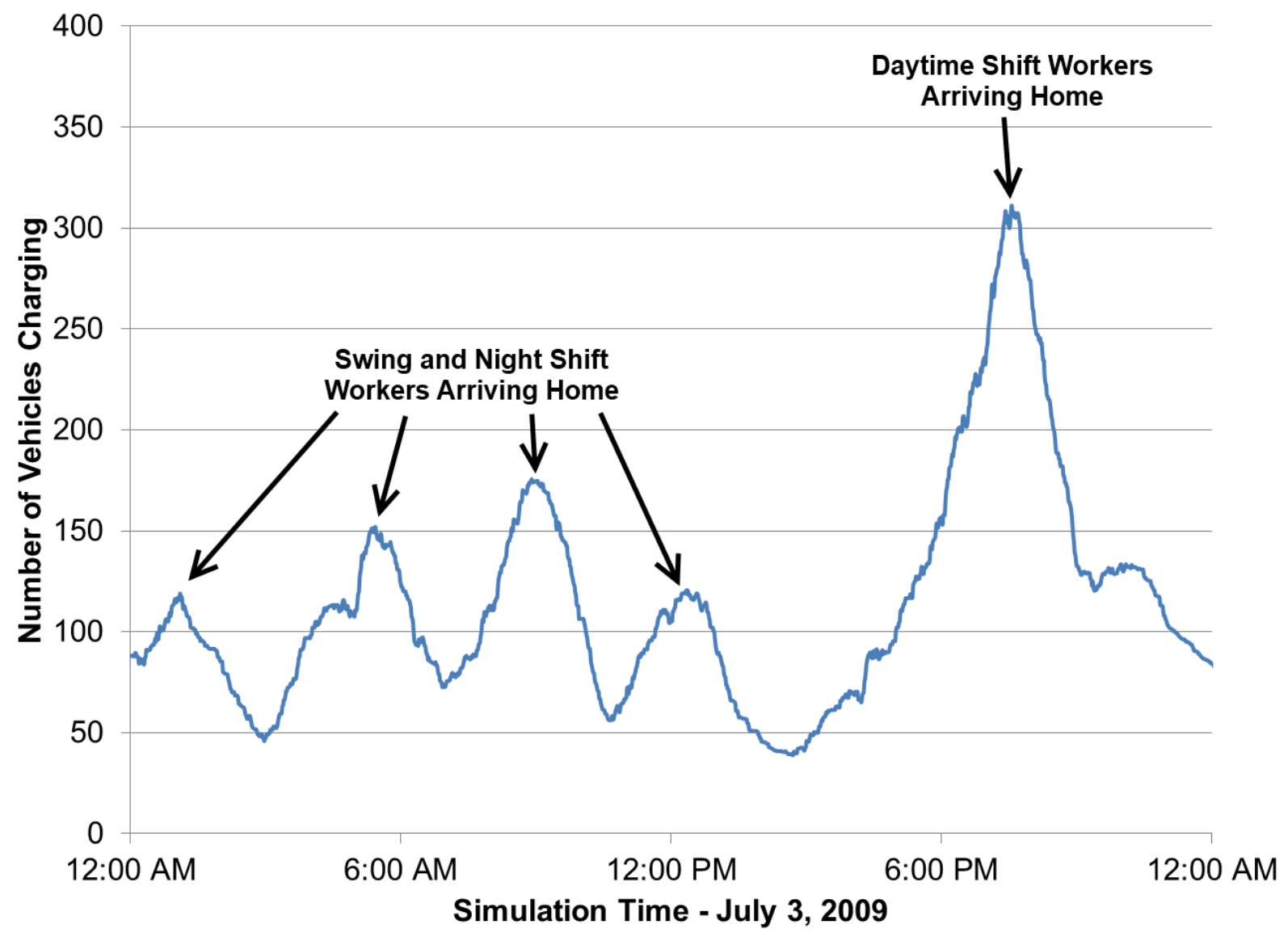

Figure 4. Number of vehicles charging during a typical simulation day

\subsection{Description of Charging}

Two different charging levels and two different charging methods or strategies were used in this study. Charging levels were representative of the infrastructure of the residence and are labeled as "Level 1" or "Level 2" (per the SAE J1772 Standard [SAE 2012]). Level 1 charging in this simulation represents a 120 VAC-connected charger that has a maximum output of $1.7 \mathrm{~kW}$. Level 2 charging is a $240 \mathrm{VAC}$-connected charger that has the ability to charge at a maximum of $7.2 \mathrm{~kW}$. It is worth noting this represents the low end of the Level 2 specification, with the J1772 standard outlining Level 2 charging up to $19.2 \mathrm{~kW}$ [SAE 2012]. While higher charging rates are part of the $\mathrm{J} 1772$ standard, the $7.2 \mathrm{~kW}$ represents a common residential 240-Volt circuit with a 30-Amp circuit breaker. For all simulation scenarios, $50 \%$ of the population was assumed to charge at Level 2 rates.

\subsubsection{Charging Methods}

Two different charging methods were also employed in the simulation. The first was a constant demand, or "unmanaged", charging. If the vehicle was in the unmanaged charging mode, it would begin charging at its maximum charge rate as soon as it arrived home and continue at the maximum rate until a full state of charge was reached. Once a full state of charge was achieved, the vehicle effectively disconnects from the grid and no longer draws power. 


\subsubsection{Grid Friendly Charging: frequency-based}

The other charging method, called Grid Friendly ${ }^{\mathrm{TM}}$ Charging, adds some intelligence to the vehicle charging. Utilizing measures of local grid conditions and stress, the Grid Friendly Charging Controller (GFC) adjusts the power consumed by the charger. If the grid is under higher stress conditions, the GFC will reduce charge rates to help alleviate the stress. With information on the charging time remaining and the level of this stress, the GFC will adjust the charge rate between no output and maximum output.

Two different grid metrics can be monitored by the GFC to evaluate "grid stress" and adjust charging power. The first method, and the method used in the previous wind-integration study, utilizes grid frequency information [Tuffner and Kintner-Meyer 2011]. The grid frequency was created by taking the additional balancing requirements associated with the addition of $10 \mathrm{GW}$ of wind generation and using the equation

$$
\frac{\Delta P}{D}=\Delta f
$$

where $\Delta P$ is the power change from the balancing signal, $D$ is a load-damping constant, and $\Delta f$ is the frequency change [Kundur 1994]. For this study, $D$ is assumed to be $94.74 \mathrm{GW} / \mathrm{Hz}$. A sample interval of the generated frequency information is shown in Figure 5. When grid frequency drops suddenly, this is an indication of either decreased generation or increased load on the system. When the GFC detects this, the charge rate is reduced. If the frequency increases, a generation increase or load decrease occurrs, so the GFC commands a higher charging rate to help stabilize the power system.

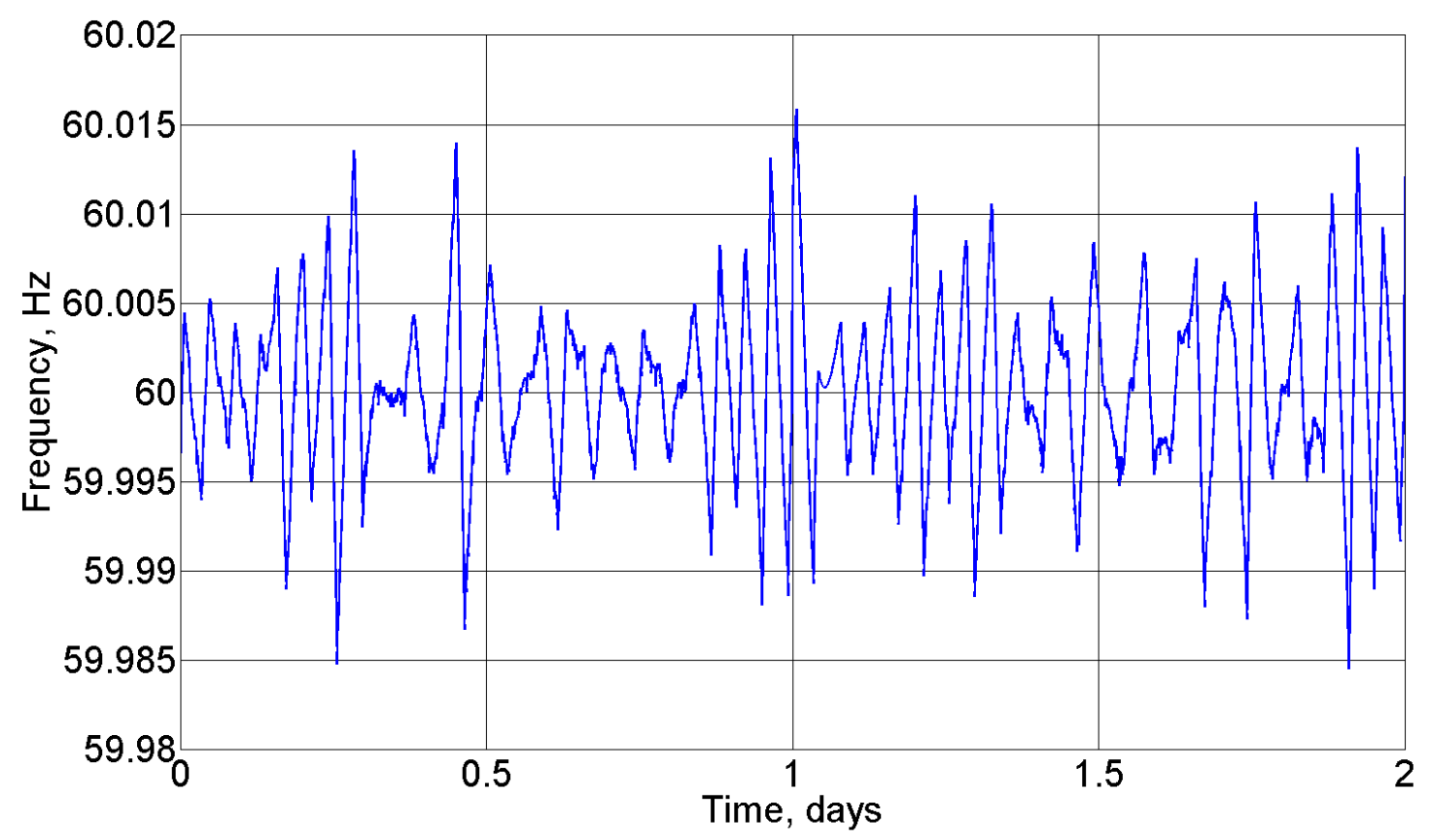

Figure 5. Sample frequency input for simulated system 


\subsubsection{Grid Friendly Charging: Voltage-based}

The second grid metric is local voltage measurements at the charging residence. Similar to frequency, a drop in local grid voltage can be an indication of increased load on that part of the system. A decrease in local grid voltage can be an indicator of increased loading and stress on the system. Under this condition, the GFC would reduce PEV charging to help stabilize the system. If the voltage rises significantly, it can be an indication of excess localized generation (e.g., a cloud transient clearing on a PV array, or a sudden drop in load). Under this situation, the GFC would increase the PEV charge rate to help keep the voltage within ANSI limits. The upper and lower limits of this charging method were meant to prevent excursions into the ANSI emergency voltage range, so a low set point of 115 Volt and a high set point of 126 Volts were utilized. Adjustment of the charging rate in relation to these two set points would occur continuously and respond in a manner nearly identical to the frequency-based charging mentioned above, but with voltage as the adjustment criterion instead of frequency.

The two different "grid stress" metrics also represent two different levels of impact. The frequency metric is typically associated with the power system as a whole and represents an indication of global stability (and bulk-power stability) on the power system. Voltage, on the other hand, is a much more local phenomenon. Voltage variations can be indicative of the full power system, but are often a direct result of local loading and system operation conditions. In a real deployment, both of these would be monitored simultaneously. However for this study, frequency monitoring and voltage monitoring were considered two separate operational modes of the GFC.

\subsubsection{Vehicle-to-Grid (V2G)}

In addition to the two methods of monitoring grid stress, two methods for responding to the stress were tested. In all GFC-charging scenarios, the GFC can modify the charging power. However, some schemes also simulated the ability of the battery to be discharged back into the grid. Unlike GFC scenarios, if a vehicle still had sufficient time before it was needed, a V2G vehicle could discharge back into the grid (as appropriate). Examples of GFC and V2G for the same interval are shown in Figure 6. Both methods respond to grid stress conditions (voltage-based and frequency-based). However, the V2G capability allows the PEV to respond long after it has reached a 100\% state of charge by discharging the storage to free-up room for additional charging. For GFC, once the battery is fully charged, the vehicle can no longer provide grid services. 

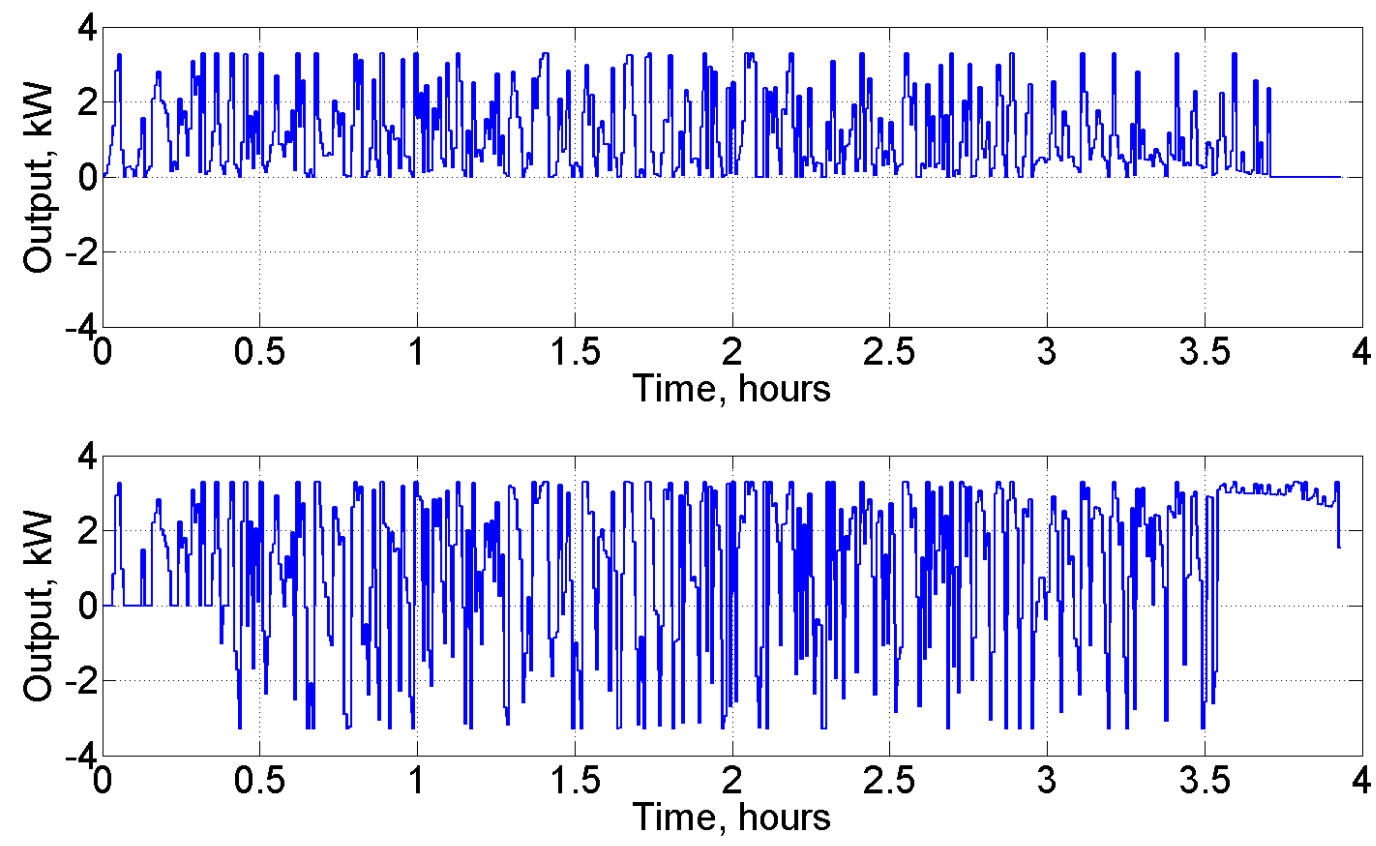

Figure 6. Example charger power for GFC (top) and V2G (bottom)

\subsection{Simulation}

Simulations were performed for a one week period during the summer. The Phoenix, AZ region and the summer season were chosen to maximize the solar generation output and its impact on the test feeder. This locational and seasonal choice emphasized the impacts of PV production. During non-summer periods, the output of the PV arrays is expected to be smaller than assessed in this study. Thus, the results could be considered as a high-stress case for the assessment of PV impacts.

During the simulation, data on various locations with the distribution feeder were monitored for various properties of the power flow. This included voltage levels at various places in the system, transformer loading, and power losses in the lines and transformers. All information was monitored in the simulation at 1-minute measurement intervals.

The data monitored are primarily directed at evaluating the impacts of photovoltaic generation and electric vehicles on two fronts: voltage and power. Voltage measurements are evaluated to examine how much time particular regions of the feeder spent outside the normal ANSI operations range of 114 VAC to 126 VAC [ANSI C84.1-2006]. The ANSI standard also dictates an emergency range of 107 VAC to $127 \mathrm{VAC}$, but this range should only be used for less than 1 minute. On the feeder simulated, Node 66 was particularly sensitive to ANSI limit excursions and will be used as the monitor point for all simulations.

Power levels were simulated to determine if photovoltaic generation or electric vehicles resulted in transformer overloading on the system. A simple transformer lifetime model was implemented. Any amount of time the transformer spent over its limit was deducted as a weighted amount from its total lifetime. A severe, short-term overload could remove as much equipment life as a very small, longer 
overload. This simplified model is based on the IEEE Standard C57.92, which estimates the reduction in transformer life as a function of thermal history of the transformer coils [IEEE, 1995].

\subsubsection{Criteria for stress conditions in distribution systems}

To evaluate the impacts of high penetrations of photovoltaics and plug-in electric vehicles on the system, two metrics of the distribution feeder were examined. The first was the feeder's ability to maintain voltage levels within ANSI standard operations range. The second was to examine the impacts on transformer loading and how the equipment life would be impacted. These metrics are described in further detail in the following subsections.

\subsubsection{Violations against ANSI voltage range}

ANSI standards established a voltage range for single-phase electricity supply. This range is defined to be between 114 and 126 Volts. Voltage regulators are used to maintain the voltage within these limits. With lack of sufficient voltage control devices or additional retro-fits of distributed generators, these limits may be exceeded. In this study, the duration in minutes and the severity of the voltage being out of range are calculated in a metric called 'volt-minute'. A 1 volt-minute indication denotes the condition in which the voltage is outside the ANSI range by 1 Volt for 1 minute.

As indicated earlier in the report, voltage measurements were collected at various point on the 123-node feeder. However, Node 66 was selected as an average, end-of-line measurement point to represent the system impacts. All voltage violation measurements are presented only for this node in the system.

\subsubsection{Transformer overload condition}

A metric for the burden on the secondary transformer life was defined that expresses the duration in hours of service life lost, called 'overloading duration'. A simplified approach was selected that counted the hours of overload weighted by the percentage of overloading. This approach captures the basics of the IEEE Standard C57.92 mentioned above, but in a simplified manner. As such, being at rated power for 1 hour uses one hour of life, while being at half-rated power for 1 hour would only use 30 minutes of total service life. This is very analogous to the volt-minutes metric described above. 


\subsection{Results}

Many different scenarios were run for a variety of photovoltaic generation scenarios, electric vehicle scenarios, and combinations of the two technologies. The results are broken down into the two metrics examined: voltage violations and transformer loading. It is important to note that the values presented in this section are for a single week of simulation in a peak solar output condition on a very specific feeder. Results will be different for other times of the year and different feeder topologies. The study conducted in this report was used to investigate some of the potential for PV and PEV integration, but is by no means an all-inclusive study.

\subsection{Overall Consideration}

While the study conducted investigated how PV generation and PEVs interact, their interactions are not always intuitive and easily predictable. One of the largest challenges with PV generation and PEV integration is their non-coincident peaks. PV generation tends to peak around noon or early afternoon, when solar flux is at its highest. Unfortunately, the peak associated with electric vehicles is typically in the late afternoon and early evening, when workers from the day shift arrive home.

The coordination of these two peaks is a significant problem for utilizing electric vehicles to integrate photovoltaic generation. The solar peak occurs during a period when many electric vehicles are not available for charging. To mitigate this problem in the simulation study, it was ensured there were members of the population that worked night (about $31 \%$ of homes) or swing (about $24 \%$ of homes) shifts, so their vehicle began charging during this solar peak. This particular consumer behavior is estimated to be higher than a typical residential neighborhood, but could represent a concentration of the population that works in fields that require such shifts (e.g., hospitals, ports, fabrication facilities).

A better solution to the PV generation peak is the inclusion of public charging stations (or work charging). The simulated feeder contains very few commercial buildings. Furthermore, the probability a person living in the area would work within the same feeder and charge their vehicle at that location is low. Regardless, the previous bulk-power study with wind integration indicated that as little as $13 \%$ of the population needs work charging ability to handle the additional balancing requirements [Tuffner and Kintner-Meyer 2011]. Such a trend may hold for solar integration as well, but the nature of the simulated feeder did not explore this possibility.

\subsection{Unmanaged Charging}

The first scenario examined utilizes the "unmanaged charging" method as the amount of electric vehicles and rooftop PV in the feeder is increased. Recall that this scenario uses a constant charge rate for the PEVs, which begins as soon as the vehicle arrives at home.

\subsubsection{Voltage}

With the varying levels of both electric vehicles and PV generation, the feeder is eventually pushed outside the acceptable operating limits of the ANSI standard. Figure 7 shows the number of minutes that Node 66 spent outside the emergency range of the ANSI voltage standard. Increasing just the number of 
electric vehicles showed no appreciable change in the violation time. However, increasing just the number of photovoltaic generators eventually ramped up to 160 volt-minutes spent in violation for the week. However, if both populations were increased, the severity of the violation went down, but was still higher for higher penetrations of solar generation.

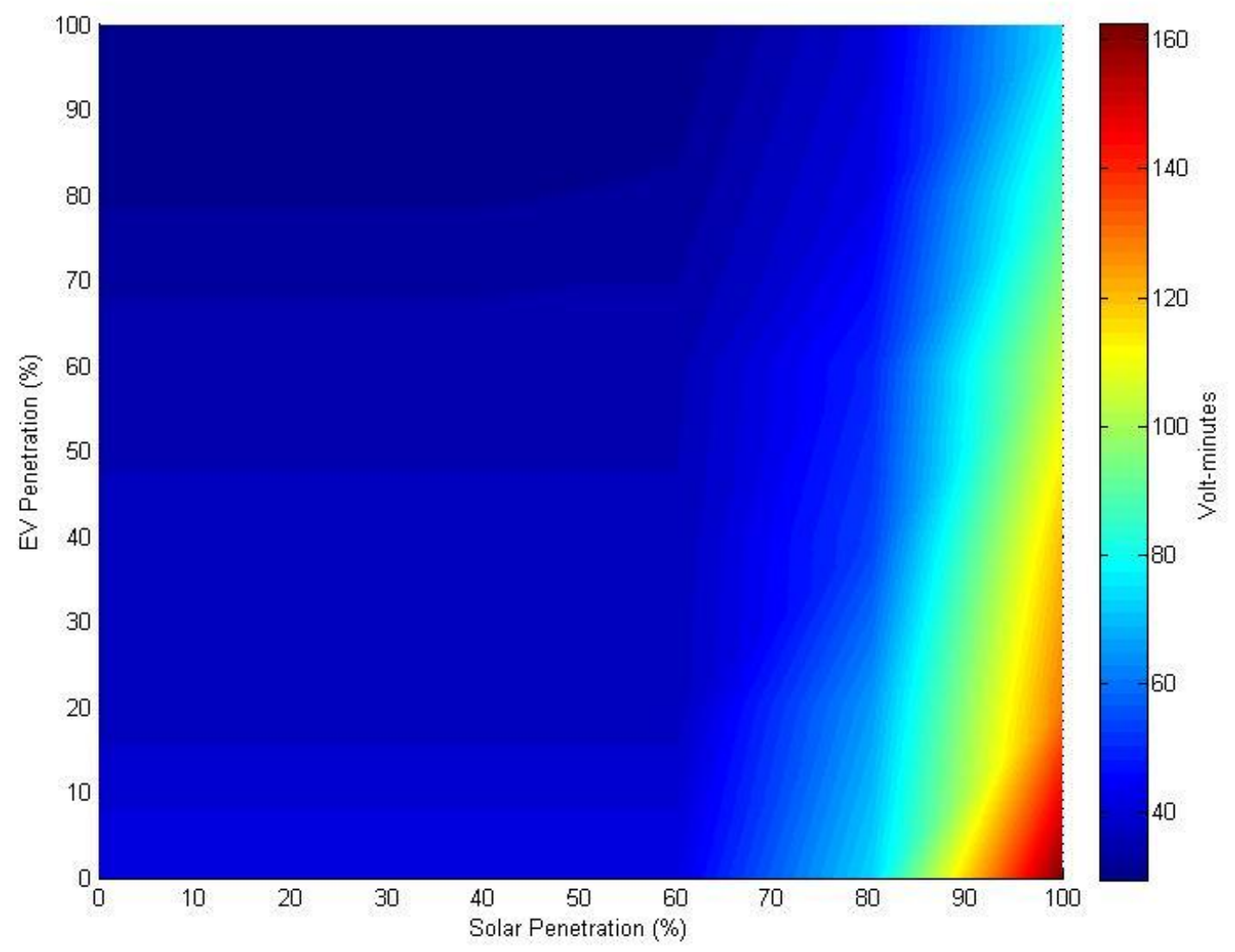

Figure 7. Time outside ANSI limits for unmanaged charging and increased PV on node 66

The results shown in Figure 7 are intuitive. As more PV is introduced into the system, it can increase the voltage at particular locations. In this case, Node 66 saw an increase in voltage as the amount of power from PV increased at that node. However, adding load to offset this increased production helped reduce the violation time. Unfortunately, the solar peak is not at the same time as the vehicle charging peak. As such, even the increased PEV population was not enough to bring the violation time down completely.

\subsubsection{Transformer loading}

The other focus in this study is the duration of transformer overloading and how much this reduced service life. Figure 8 shows the difference between the lifetime reduction and the number of hours in 1 week for a specific, representative transformer of the system. This particular transformer was selected as it represented the average behavior of all distribution transformers on the system. Positive numbers represent a transformer that was overloaded on average, and negative numbers represent the transformer being under loaded. Notice that the system starts out with the transformer being just under rated power for 
the duration of the week. As the number of electric vehicles increases, the load increases and the transformer spends more hours in an overloaded condition. However, increasing the amount of PV generation on the system serves to reduce the duration of overloading. For instance, at $60 \%$ PEV penetration, a 20\% PV penetration results in about an 18-hour reduction in life lost. An additional 20\% PV penetration (total 40\%) reduces the life lost by about another 16 hours. It can be observed that the return on lifetime reduction diminishes with higher penetration of PV.

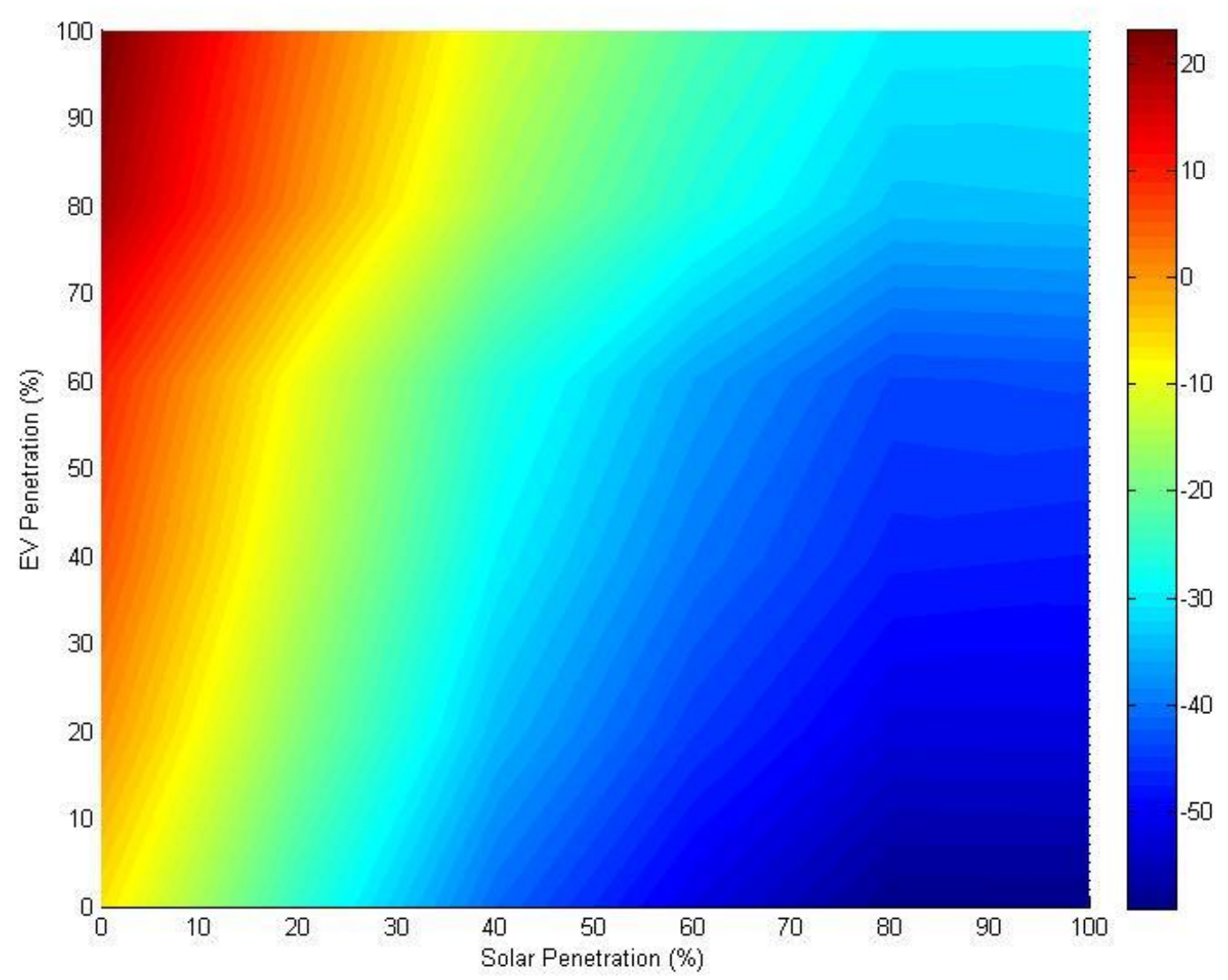

Figure 8. Hours transformer is overloaded for unmanaged charging and increased PV

Figure 8 highlights another expected behavior. As the system load is increased (with more PEVs), the time the transformer is overloaded increases. However, if PV generation is added, it helps reduce the amount of power flowing through the transformer. This serves to reduce the duration of overloading.

\subsubsection{Summary}

The introduction of unmanaged charging electric vehicles and larger numbers of photovoltaic generation affected the system in an expected manner. As load (PEVs) increased, the transformer loading increased and the voltage levels dropped. As PV array counts increased, the transformer loading decreased, but more voltage violations occurred. With a combination of the two technologies, transformer loading was reduced from the PEV-only scenario, and voltage violations were reduced from the PV-only scenario. However, the non-coincident nature of the two technologies means that even though both quantities were 
reduced, a full PEV-PV population did not fully remedy or offset the negative impacts that either technology caused.

\subsection{Frequency-based GFC}

The addition of intelligence to the electric vehicle charging allows the charging rate to respond to indications of grid stress. For the first scenario, the Grid Friendly Charging Controller is utilizing frequency as the grid stress measurement. This quantity is more in tune with global impacts on the power system, so the immediate feeder concerns may not necessarily be addressed.

\subsubsection{Voltage}

As with the unmanaged charging scenarios, the first metric to examine is voltage for Node 66 on the 123node system. Unlike the unmanaged charging simulations, the GFC-based charging was simulated with both charging only (GFC) and charging/discharging (V2G) control.

\subsubsection{GFC}

With only the ability to vary charging rates, the voltage violations shown in Figure 9 closely match those of the unmanaged charging results in Figure 7. Increased PV generation still results in nearly 160 voltminutes spent in violation for the week, and an increased PEV population helps reduce this time, but not eliminate it. This result is very similar to that shown in Figure 6. The key reason for that is that the global grid-frequency is not a good predictor for local voltage problems in the distribution system. 


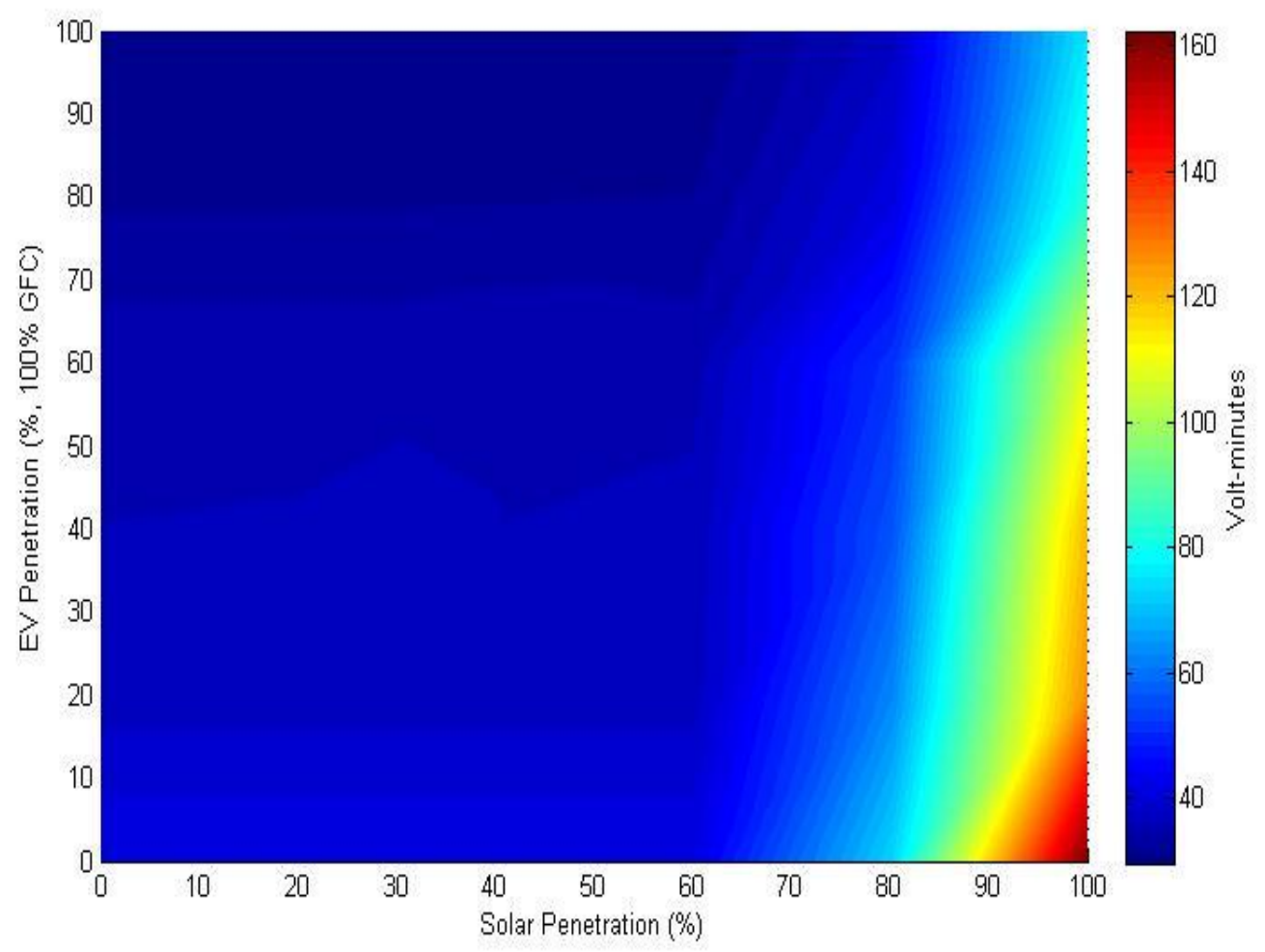

Figure 9. Time outside ANSI limits for GFC frequency-based charging and increased PV

\subsubsection{V2G}

Incorporating the ability to discharge the battery changes the results significantly. The amount of time spent in violation for the V2G case is shown in Figure 10, and it is about 11 times the peak value from Figure 9, as indicated by the much larger color scale on the figure. Increasing the number of electric vehicles and the number of PV arrays both increased the time spent in voltage violation. The combination of both resulted in nearly 1800 volt-minutes of operation outside the ANSI emergency voltage range. 


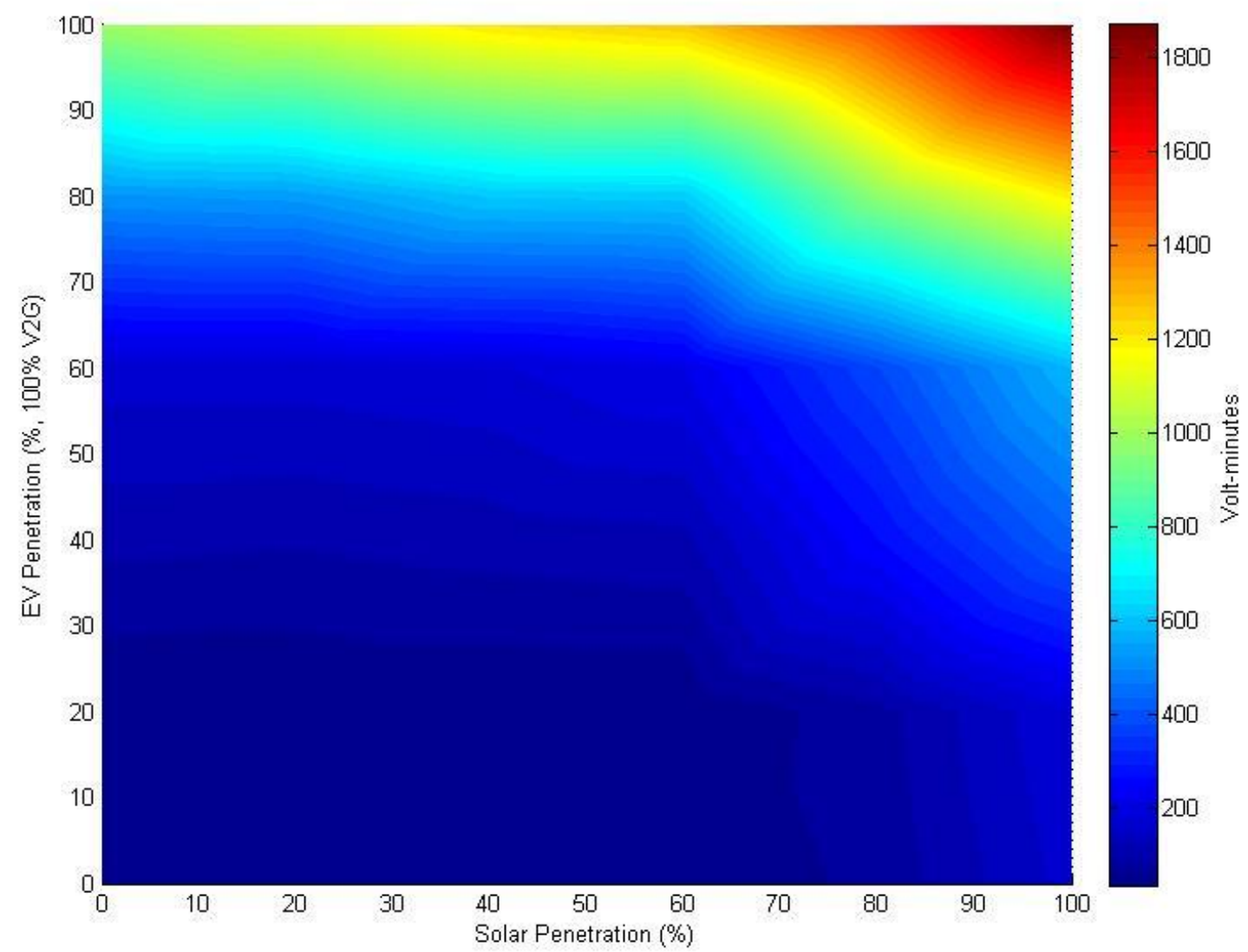

Figure 10. Time outside ANSI limits for V2G frequency-based charging and increased PV

The results of Figure 10 are a little confusing at first. The increased amount of PV certainly is expected to increase the voltage and could result in more minutes spent outside the emergency voltage range. Adding additional $\mathrm{V} 2 \mathrm{G}$ vehicles only exacerbates this problem. Because the V2G technology sometimes acts as a generator, it raises the voltage even further than the PV alone, causing further violations. A voltage-based charging control method is explored in a later section to examine mitigations to this impact.

The results for this method of GFC charging are overall transmission system-centered and use frequency as the metric for charge rate adjustment. As shown in the example plot of Figure 5, the frequency deviates both up and down throughout the day. While charging the battery results in a net consumption of energy, the lower frequency periods result in the V2G vehicles adding to the PV arrays' generation output. While this is helping the overall system (on a bulk-power level), it is actually increasing the voltage problems associated with the local distribution feeder.

\subsubsection{Transformer overloading}

Along with the voltage considerations, the transformer loading effects of the GFC frequency-based charging are also of interest. Both the charge-only (GFC) and charge/discharge (V2G) capabilities were again simulated. 


\subsubsection{GFC}

As with the voltage violations plot above, the amount of time the transformers of the system spent overloaded under the GFC frequency-based charging looks nearly identical to the unmanaged charging scenario presented earlier in Figure 8. Figure 11 shows the increase of PEVs resulted in more time spent overloaded, but the introduction of photovoltaic generation reduced the overloaded time. As with the unmanaged charging scenario, the lowest amount of overage time is with a full PV generation population and no electric vehicles.

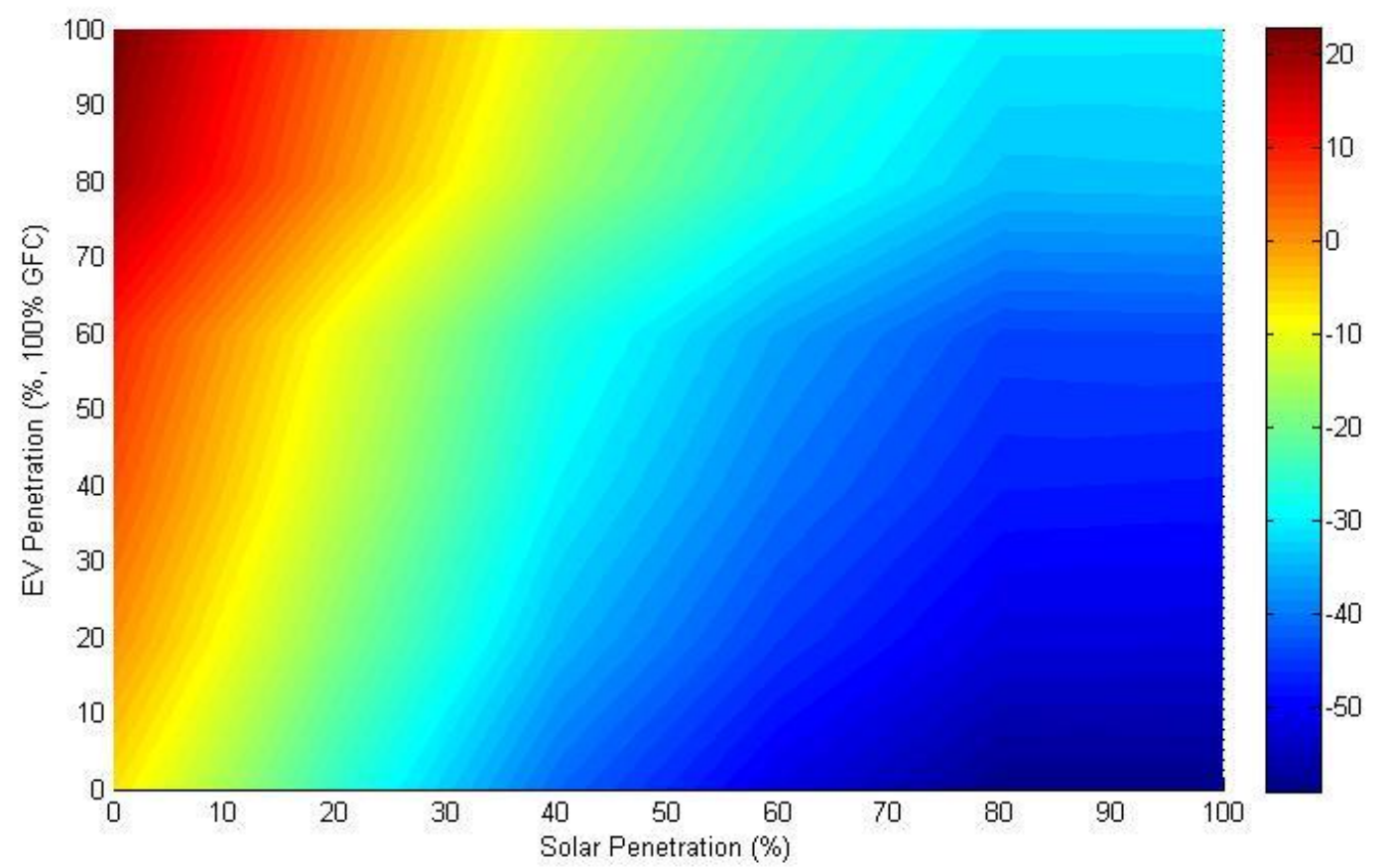

Figure 11. Hours transformer is overloaded for GFC frequency-based charging and increased PV

\subsubsection{V2G}

Similar to the voltage results presented, the transformer loading with the frequency-based V2G charging is significantly different than the GFC and unmanaged charging scenarios. As Figure 12 demonstrates, the introduction of V2G-based PEV charging results in a larger amount of time spent overloaded, with a smaller population. The upper end of the color scale for Figure 12 increases by 30 hours. The introduction of photovoltaic generation helps to alleviate the overloading condition. However, a full PEV/full PV population still has transformer overloaded hours. 


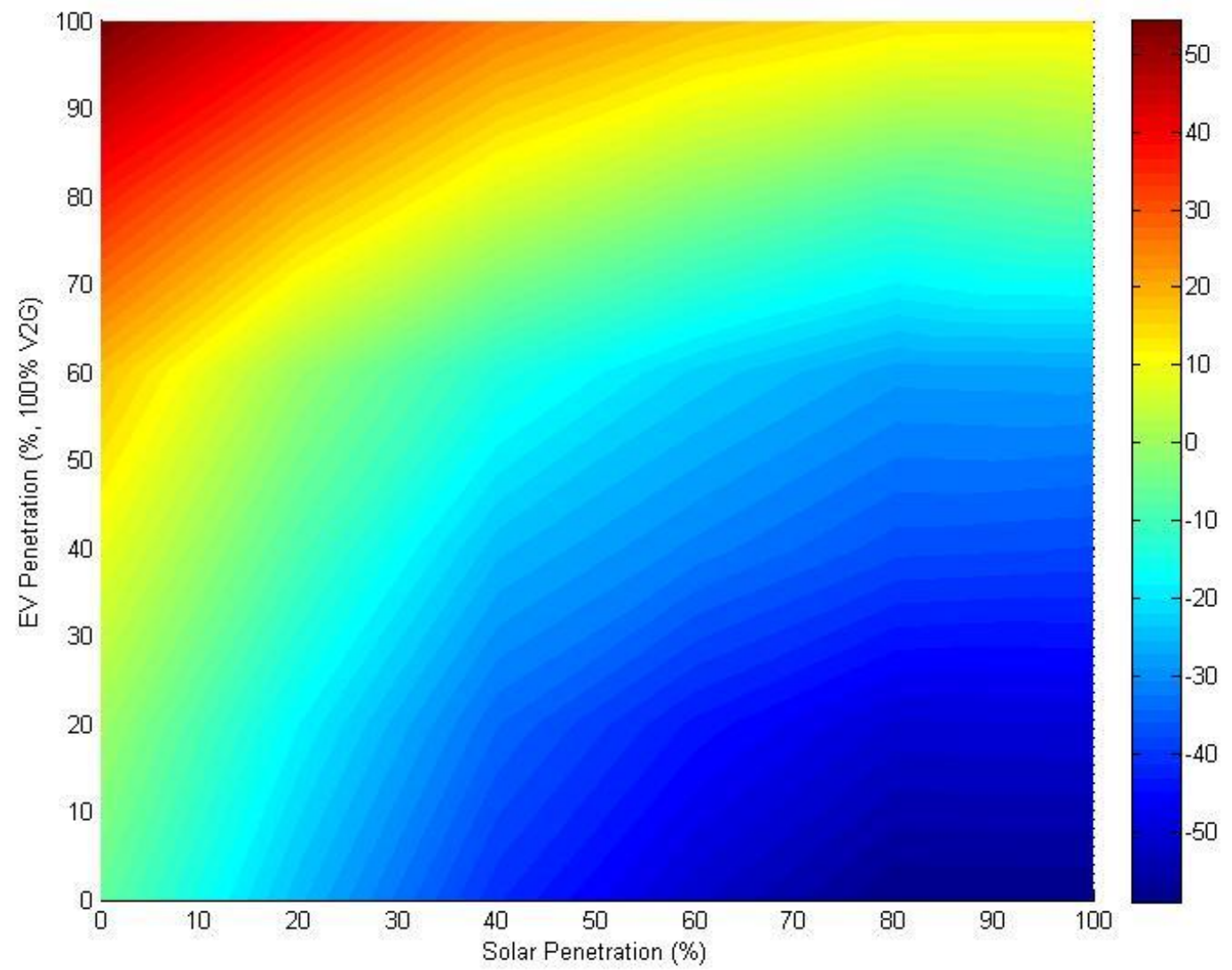

Figure 12. Hours transformer is overloaded for V2G frequency-based charging and increased PV

Similar to the voltage excursions above, the V2G-frequency based charging increases the time spent in a detrimental condition. As explained before, the frequency-based Grid Friendly Charging is adjusting the charge rate to benefit the power system on a larger level, the bulk transmission system. In the process of doing this, it is actually causing more harm to the local distribution grid. With the frequency-based control, the PEVs are often acting as supplemental generators during periods of high solar output. This has the ability to push the transformer into an overload condition while pushing the power the opposite direction (up the feeder instead of pulling it down the feeder). However, discharging the battery for this condition will require a larger charging current later to offset the lost capacity and enable the PEV to reach full state of charge by the time the vehicle is needed again. As a result, it increases the loading during already peak times and makes the overloaded condition last longer.

\subsubsection{Summary}

The frequency-based Grid Friendly Charging methods yielded mixed results. The GFC implementation did not show any significant change in voltage violation time or transformer overloading compared to the unmanaged charging scenarios because the local voltage in the distribution system is independent of the grid frequency. However, the V2G-based charging showed significant increases in voltage violation times and transformer overloading periods because this strategy injects power into the system at times, which exacerbates voltage control under high voltage conditions. Overall, frequency-based charging methods 
did not show any benefits on the distribution system. V2G application with a frequency-control strategy could have negative impacts under high voltage conditions.

\subsection{Voltage-based GFC}

To adjust the charging strategy to local conditions, the GFC charging strategy was modified to the voltage-sensitive control strategy. Only the V2G-based GFC scheme is examined for voltage-based control because it provides the most flexibility for influencing conditions both above and below the ANSI voltage limits.

\subsubsection{Voltage}

With the voltage-based Grid Friendly Charging Controller implemented, the amount of time spent outside the ANSI emergency voltage range is, for the most part, increased slightly. Comparing Figure 7 and Figure 13 the largest difference appears near the right edge of the figure (representing maximum solar penetration). While most of the cases show nearly identical results (within a few percent), one case shows a noticeable decrease. At the $100 \%$ solar, $80 \%$ PEV case, a nearly $15 \%$ reduction in volt-minute violation time is observed. Overall, the voltage-based V2G charging is providing little beneficial or detrimental impact to the distribution feeder voltage.

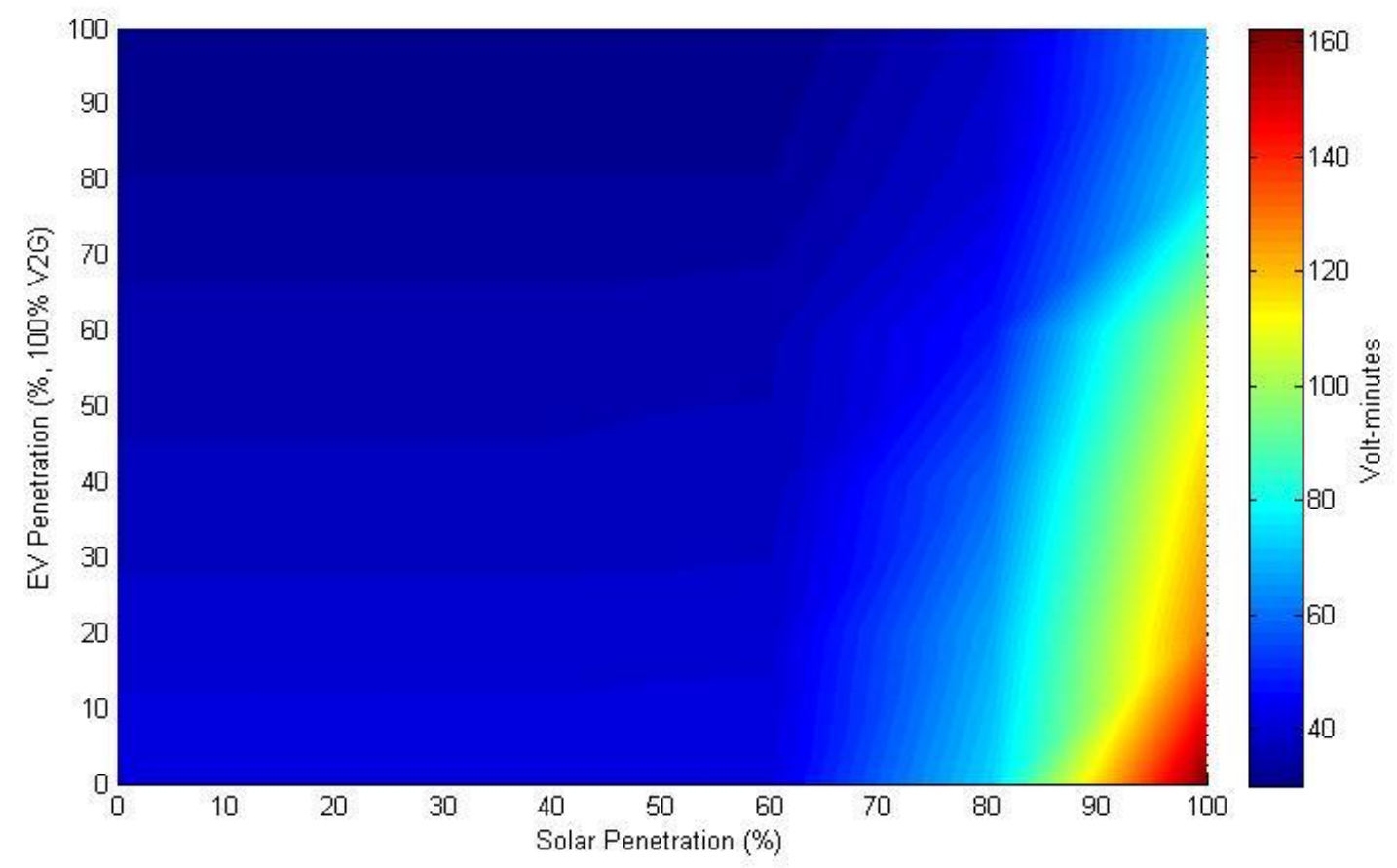

Figure 13. Time outside ANSI limits for V2G voltage-based charging and increased PV

During periods of high solar output, the voltage-based GFC will increase the charge rate of the vehicle to compensate. During intervals of low voltage and low solar output, the PEV can actually discharge into the system to stabilize the local voltage. The combination of these two capabilities theoretically allows the PEVs to integrate solar generation with fewer voltage violations. However, the results in Figure 13 show 
little overall impact, except for one specific scenario region. Obviously, set point and charging behavior changes to the voltage-based algorithm could impact the amount of time spent in the violation region. This may also be a specific observation to Node 66, but not elsewhere on the system. Availability of the PEVs (based on the work schedule of the owners) will also influence this result. Regardless of the influencing factors, overall the impacts to the voltage violations were modest for voltage-based V2G charging. Only a specific solar and PEV combination showed a benefit, with a reduction of about $15 \%$ of the voltage violation severity. For other combinations, the voltage-based V2G had little impact.

\subsubsection{Transformer overloading}

While the voltage-based GFC scheme is examining local grid stress to minimize PEV impact on the distribution system, it may not necessarily reduce the transformer overloading periods. However, Figure 14 shows the local grid benefits extend to transformer overloading as well. Much like the time spent exceeding voltage limits, the time spent in overloading conditions is only marginally impacted for voltage-based GFC. Figure 14 looks structurally very similar to the unmanaged charging scenario of Figure 8 , with the actual overloading hours being nearly identical for all scenarios.

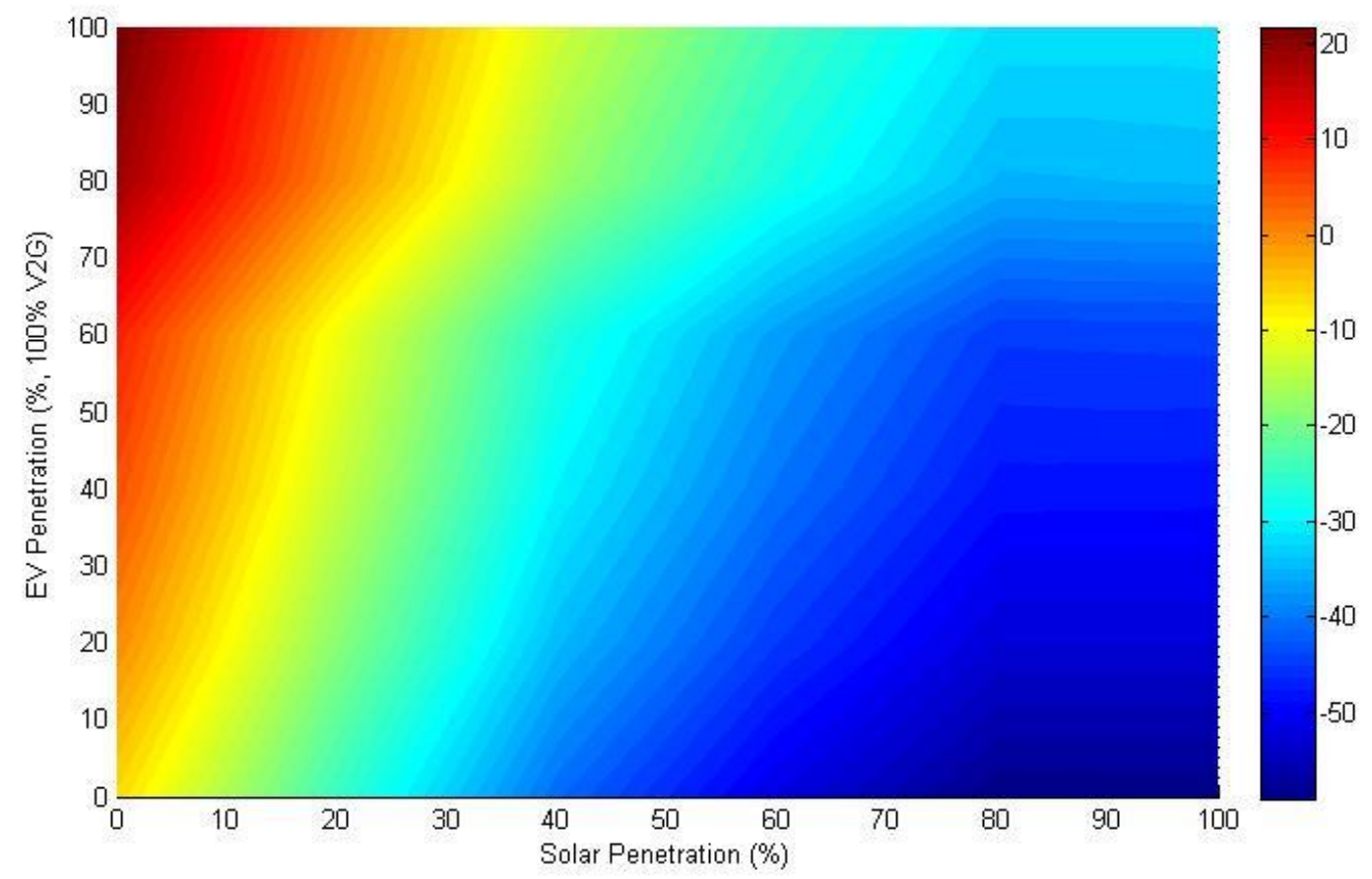

Figure 14. Hours transformer is overloaded for V2G voltage-based charging and increased PV

While the voltage-based GFC charging is trying to influence only voltage levels, it will indirectly influence the transformer loading. With the V2G capability, it can supplement solar shortfalls and help reduce the overall loading on the transformer. Furthermore, adjusting the charge rate based on voltage will inherently shift the charging to periods of lower load, such as those in the early morning. However, as with the voltage violation results in the previous subsection the voltage-based charging had little overall impact on the transformer overloading. Contrary to the voltage violations, there does not appear to be a specific case that shows a noticeably different impact. 


\subsubsection{Summary}

Voltage-based Grid Friendly Charging provided little change to the distribution system over the unmanaged charging schemes. Differences between the two schemes were on the order of a few percentage points and not significant. Much of this minimal impact is attributed to generic set points for the voltage-controlled method, as well as overall availability problems. With the upper and lower limits of the voltage-based $\mathrm{V} 2 \mathrm{G}$ charging fixed, the system only drastically responded when the system was nearing a voltage violation. While this had some minor impacts on the voltage violation severity, it had little impact on the transformer loading. Time-varying limits, or tuned limits, for individual charging devices could improve the local distribution impacts.

The larger influencing factor is the overall availability of the PEVs during periods of higher PV output. Even with the extra night shift and swing workers described earlier in the system, not enough PEVs were available during periods of larger solar output to create a significant impact on the system. However, despite voltage-based PEV charging providing little impact on the local distribution network stress, it did not detrimentally influence it like the frequency-based charging. By examining local metrics of the system to influence the charging, the voltage-based charging limited detrimental impacts.

\subsection{Overall Observations}

The inclusion of high penetrations of electric vehicles and solar photovoltaic generation had mixed impacts on the test distribution feeder. Increasing the number of vehicles increased the overall load of the system, which typically resulted in greater burden on the distribution system components, which are manifested in greater frequency and longer transformer overload periods. Increasing the PV arrays typically increased the burden on the voltage regulation, as expressed in duration spent above the ANSI standard upper limit of 126 Volt. The combination of the two technologies yielded positive and negative results, depending on the scale of the penetration of each technology.

\subsubsection{PEV and PV can work synergistically together}

Without applying any charging strategies (unmanaged charging), PEV and PV are often working synergistically to a certain degree. We found that in a highly loaded distribution system, PV can offset the additional burden imposed by PEVs on transformer loading. This result is intuitive because PVs were assumed to be supplying electricity downstream through secondary transformers. The improvement in reduced transformer overloading was about $11 \%$ when $20 \%$ of the homes were equipped with PV arrays at a 50\% PEV penetration scenario. Additional PV penetration achieved diminishing returns on overloading improvements.

For voltage control problems, the study indicated significant sensitivity to high penetrations of PV rooftop technology. The simulation showed no impacts on voltage control up to about $60 \%$ of penetration. At higher levels of PV penetration, the metric increased to 160 volt-minutes within a summer week. The voltage control problems were all associated with high voltage conditions, exceeding the upper limit of the ANSI voltage range. The additional load of PEVs is then reducing the voltage in the feeders and thus improving the overall voltage control. However, because of the non-coincidence between PEV charging and PV electricity output, the mitigation effectiveness is relatively weak. At a 100\% PV penetration, the 
metric of voltage violation can be reduced from 160 volt-minutes to 110 volt-minutes at a PEV penetration of $50 \%$ and to about 70 volt-minutes at $100 \%$ PEV penetration (see Figure 7).

\subsubsection{Impact of charging control strategies and V2G applications}

Two charging strategies and two V2G applications were investigated. The inputs for the charging strategies were: 1) frequency deviations for one set of charging strategies, and 2) voltage measurement for the other. The control variable was the transfer of power, which could be regulated between 0 and $100 \%$ of charging current.

Figure 15 summarizes the results for the different charging, PEV penetration, and PV penetration levels for a given transformer overloading scenario. In this particular case, the penetration levels associated with no increase in transformer overload time are examined. That is, for that combination of photovoltaics and electric vehicles, the transformers did not exceed the expected life loss for operating at $100 \%$ their rated power for the entire simulation duration. In Figure 15, any combination below the plotted lines is a valid $\mathrm{PEV} / \mathrm{PV}$ penetration pair that will result in normal or lower rates of life loss in the transformer. The unmanaged charging, GFC-frequency, and V2G-voltage strategies all can support a 100\% PEV penetration with about $40 \%$ solar (V2G-voltage actually requires a marginally smaller solar penetration at about $37.5 \%$ ). The overall key message is that the frequency GFC and voltage-based V2G strategies do not significantly improve the burden on the transformer loading. The voltage-based charging strategy indicated the best overall improvement, although an inconsequential amount.

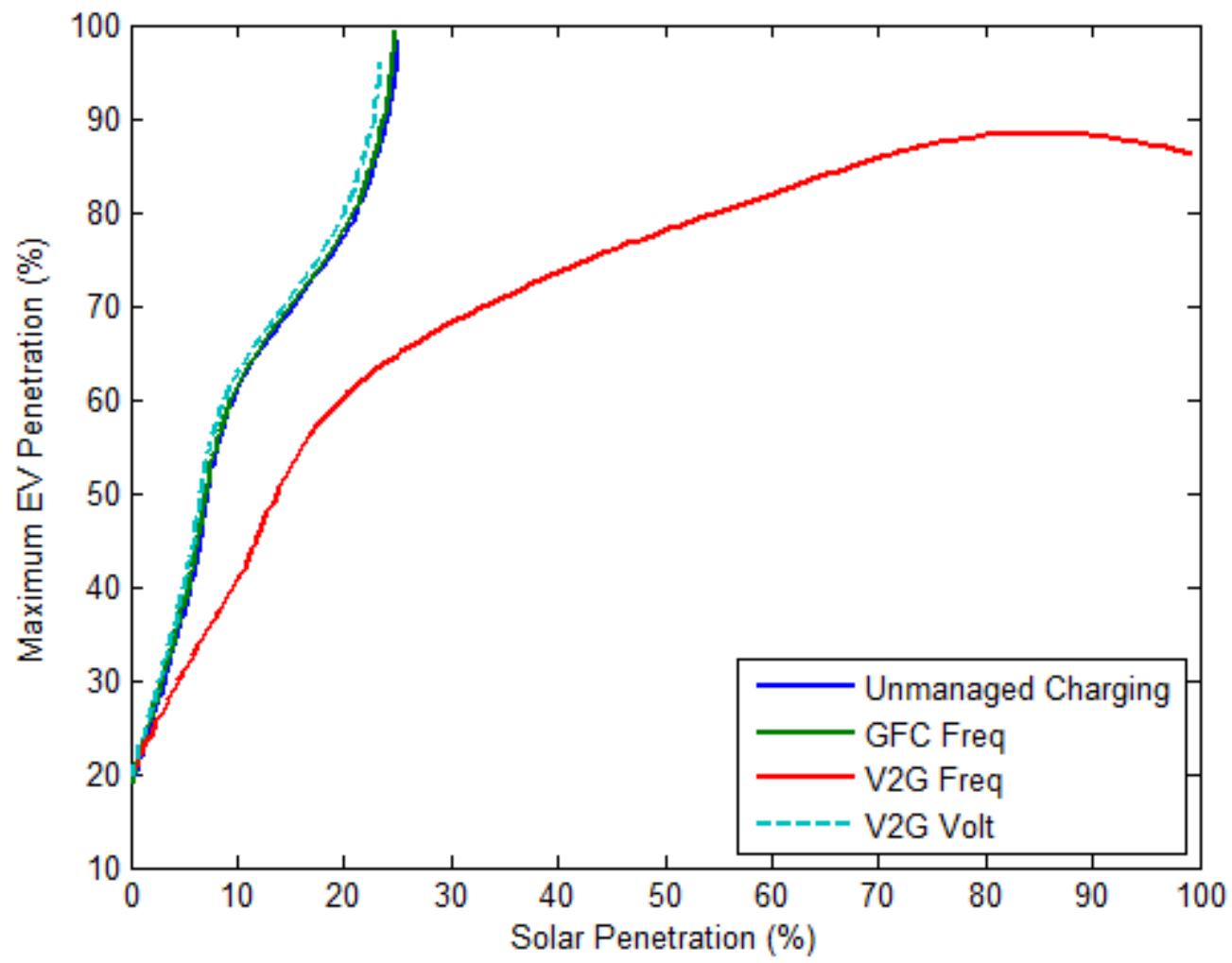

Figure 15. Maximum PEV population for given solar population and no additional transformer overloading 
It should also be mentioned that a V2G strategy could exert an additional burden on a highly loaded distribution system when it is controlled to meet transmission system objectives, such as primary frequency control or regulation services, which are generally independent of local voltage or loading conditions. The V2G-frequency-based strategy in Figure 15 indicates that the overloading conditions increases with growing PEV penetration.

\subsubsection{ANSI voltage range violations}

Of interest is the question of how much of an improvement in the voltage control could be achieved by having a voltage-based PEV charging strategy employed as opposed to the unmanaged PEV charging? Comparing Figure 7 and Figure 13 suggests that any improvement is minor. At high PV penetration, the voltage-based control strategy indicates some reduction in the voltage range violations, which were related to exceeding the voltage limits of 126 Volts. Although, the voltage-based charging strategy targeted a relevant control variable in the distribution system, the lack of coincidence between the PEV resource availability and the high grid stress rendered this method only partially effective.

The frequency-based charging strategy had no effect on the voltage-control quality in the distribution system. This result is attributable to the fact that frequency is an overall transmission system property and does not reflect localized voltage issues in distribution feeders.

\subsubsection{Transformer overloading}

Using the voltage-based control strategy had a very minor effect on the overloading conditions of the secondary transformer. This result is defensible, because the voltage control scheme did very little to reduce to overall load for PEV charging. It was primarily sensitive to voltage conditions at the high and low end of the ANSI voltage range, which resulted only in a few hours of load reduction due to high or low voltage conditions.

The frequency-based charging GFC strategy also had very little impact on the overloading conditions of the transformers. This was somewhat surprising because the overall charging rate is reduced to accommodate some variability in the charging rate about an operating point that is less than the full rate. However, the frequency data utilized does not necessarily support this trend. Recall Figure 5, which showed a sample of the grid frequency for the simulation. The frequency-based charging methods utilize a 5-minute moving average to determine charge rates in relation to frequency. As Figure 5 shows, there are many "gradual uptrends" in the frequency, but most of the downward shifts are quite fast. What this means is the frequency-based charging will be consistently biasing itself to slightly higher charge rates (to absorb excess generation), except for period drops where it will turn off the charger or discharge into the grid. If the frequency characteristics of the simulation interval were smoother or more symmetric in the ramp up and ramp down periods, it is expected the frequency-based GFC and V2G charging methods would have shown a larger impact. 


\subsection{Conclusion}

This analysis provides some initial insights into the leveraging potential of distributed PV and PEV charging. Either of the two technologies by themselves - at some high penetrations - may cause some voltage control challenges or overloading problems, respectively. But when combined, there - at least intuitively - could be synergistic effects, whereby one technology mitigates the negative impacts of the other. High penetration of PEV charging may overload existing distribution system components, most prominently the secondary transformer. If PV technology is installed at residential premises or anywhere downstream of the secondary transformer, it will provide another electricity source thus, relieving the loading on the transformers. Another synergetic or mitigating effect could be envisioned when high PV penetration reverses the power flow upward in the distribution system (from the homes upstream into the distribution system). Protection schemes may then no longer function as designed and voltage violation (exceeding the voltage upper limited of the ANSI voltage range) may occur. In this particular situation, PEV charging could consume the electricity generated from the PV, such that the reversal of power flow can be reduced or alleviated. Given these potential mutual synergistic behaviors of PV and PEV technologies, this project attempted to quantify the benefits of combining the two technologies. In addition, we explored a set of PEV control strategies and their impacts on the overall synergetic opportunities.

This is one of the initial studies, if not the first study, that explores the synergies between distributed PV and PEV charging. To set the right expectation for the outcome of this study, the authors would like to point out that the parameter space for this analysis is very large, requiring literally thousands of scenarios of different distribution system topologies and loadings on individual feeders and segments of the distribution system. Just the vastness in diversity of distribution system topology and customer composition in the system, plus the diversity in distribution system design guidelines that recommend sizing of distribution components spans a very large parameter space that this study cannot address in its entirety. Only a small piece of this much larger analysis 'picture' can be addressed in this report because of limited funding resources. We chose one and only one distribution system (IEEE 123-node system) and made assumptions regarding the existing loading for the end-user nodes. Furthermore, we selected a U.S. southwestern location (Phoenix, AZ) to maximize the solar electricity output by the distributed PV arrays. Overall, within reasonable selection ranges, we created a scenario that was certainly favorable to high PV electricity output and high overall loading in the entire distribution feeders. Because of the very targeted scope of this analysis (one feeder design, one location), the authors caution against extrapolating the results outside the context of the scenario definitions and underlying assumptions. The results should be considered as favorable for PV impact because of the high solar insolation in the Southwestern region. Therefore, the impacts of PV may be considered optimistic compared to regions with less solar insolation, such as the Midwestern and East Coast regions.

Overall, the severity of impacts both on the PV and on the PEV side was rather small in this study. Impacts to the distribution system occurred at higher levels of penetration of either technology. However, the penetrations had to reach above $50 \%$ to reveal noticeable impacts. The mitigating ability of one technology to absorb the impacts of the other was only minor. The results did indicate that PEV charging mitigates high voltage conditions and that overloading conditions can be reduced with onsite PV 
generation. However, it requires significant penetration of the other technology to show a mitigating effect.

Regarding the charging control strategies, we investigated four strategies: 1) unmanaged or uncontrolled charging that assumed the vehicle will initiate the charging once it arrives at home, 2) frequency-based charging, which controlled the charging current as a function of frequency deviation from $60 \mathrm{~Hz}$. In cases of over-frequency $(>60 \mathrm{~Hz})$, the charging current would increase from an operating point of charging. In cases of under-frequency $(<60 \mathrm{~Hz})$, the charging current would decrease from its operating point. 3) voltage-based charging. The control sequence increases the charging from an operating point during high voltage condition and reduced or stopped the charging during low-voltage conditions. 4) vehicle-to-grid (V2G) control strategies in which electricity could flow bi-directionally in and out of the battery.

The frequency-based charging strategy simulates transmission-related conditions, which in most cases are decoupled from local voltage conditions in the distribution system. As a consequence, the results indicated no improvements in the voltage violation with a frequency-based strategy. In fact, because of overall load-reducing effect that all strategies have, the voltage mitigation solution was less effective than the uncontrolled or unmanaged charging strategy. V2G strategy tended to exacerbate the problem of high voltage conditions. Because the $\mathrm{V} 2 \mathrm{G}$ injects electricity back into the grid, the high voltage condition problem under high PV penetration was worsened. This result has some note-worthy implications. V2G applications have been contemplated in the context of providing regulation services for the bulk-power system. Under some circumstances, V2G can be detrimental to the local conditions if there are some high voltage issues. These issues were not observed for PEV smart charging (frequency-based or voltagebased), because the PEV charger would never be turned into a generator.

In summary, key outcomes of this target analysis are:

- $\quad$ PEV and PV can operate synergistically in distribution system. PEV charging can provide additional load under high voltage conditions to reduce the excess voltage. PV resources provide an additional power injection mitigating overload conditions on secondary transformers under high PEV assumptions.

- The leveraging sensitivity was analyzed to be low, meaning that it would take significant amounts of the mitigating technology penetration to achieve an appreciable effect. However, the used 123node feeder is relatively "stiff" with relatively short distances and low impedance between nodes. Distribution systems with long feeders may show a higher sensitivity with respect to voltage deviations and therefore the synergy between PV generation and EV charging may be more pronounced.

- The PEV charging strategies showed mixed results. The frequency-based PEV charging strategy revealed the decoupled effect between the distribution system dynamics and that of the bulkpower system. The voltage-based control strategy showed improved results, since it was sensitive to changing local voltage conditions. V2G could be detrimental to high voltage conditions in distribution system feeders because it can turn an electric vehicle into a generator. PEV smart charging (frequency or voltage-based) showed only marginally improved results over the unmanaged charging. 
- The big caveat of this analysis was these results are only applicable for the 123-node IEEE feeder with some assumptions. It is difficult to extrapolate these insights to other distribution feeders in a particular service territory, as many of the assumed conditions, such as distribution system topology, customer composition, and overall component sizing guidelines vary over a wide range.

- Furthermore, another caveat is that some of the PV inverter technology may in the future be equipped with a four quadrant Volt/Var control, which may provide sufficient voltage controllability in the PV inverter. If each PV array or each PV installation could be equipped with such a smart inverter, voltage issues in the distribution system may largely be solved. 


\subsection{References}

American National Standard Institute (ANSI), “American National Standard for Electric Power Systems and Equipment - Voltage Ratings (60 Hz) - Standard C84.1-2006,“ January 30, 2012 ,Accessed May 17, 2012. [Online]. Available: http://www.nema.org/stds/c84-1.cfm

Center for Climate and Energy Solutions (C2ES), "Renewable and Alternative Energy Portfolio Standards," May 16, 2012, Accessed May 16, 2012. [Online]. Available: http://www.c2es.org/what_s_being_done/in_the_states/rps.cfm

GridLAB-D, [Online] Accessed May 28, 2012, http://sourceforge.net/projects/gridlab-d/

Institute for Electrical and Electronic Engineers (IEEE) Distribution Planning Working Group, "Radial Distribution Test Feeders," IEEE Transactions on Power Systems, vol. 6, no. 3, pp. 975-985, August 1991.

Institute for Electrical and Electronic Engineers (IEEE), "IEEE Guide for Loading Mineral Oil-Immersed Transformer". IEEE Std. C57.91. 1995.

Kintner-Meyer, M. C. W., K. Schneider, Y. Zhu, and R. Pratt, "Impacts Assessment of Plug-in Hybrid Vehicles in Electric Utilities and Regional U. S. Power Grids, Part 1: Technical Analysis," PNNL-SA-52337, Pacific Northwest National Laboratory, Richland, WA, January 2007.

Kintner-Meyer, M. C.W., P. Balducci, C. Jin, T. Nguyen, M. Elizondo, V. Viswanathan, X. Guo, and F. Tuffner, "Energy Storage for Power Systems Applications: A Regional Assessment for the Northwest Power Pool (NWPP)," PNNL-19300, Pacific Northwest National Laboratory, Richland, WA, April 2010.

Kundur, P., Power System Stability and Control, McGraw-Hill, Inc., New York, NY, 1994.

Makarov, Y., M. Kintner-Meyer, P. Du, C. Jin, and H. Illian, "Using DFT for Cycle Analysis of Energy Storage Capacity Requirements to Accommodate High Penetration of Renewable Resources in the WECC System," in Proceedings of the IEEE PES Innovative Smart Grid Technologies Conference, Gaithersburg, MD, Jan. 19-21, 2010.

National Oceanic and Atmospheric Administration, "Land-based Datasets". Accessed March 3, 2012. [Online]. Available: http://www.ncdc.noaa.gov/land-based-station-data/land-based-datasets.

National Renewable Energy Laboratory, "National Solar Radiation Data Base - 1961- 1990: Typical Meteorological Year 2," Accessed March 3, 2012. [Online]. Available: http://rredc.nrel.gov/solar/old_data/nsrdb/1961-1990/tmy2/.

Singh, R. and B. Vyakaranam, "Evaluation of Representative Smart Grid Investment Grant Project Technologies: Distributed Generation,” PNNL-20792, Pacific Northwest National Laboratory, Richland, WA, February 2012. 
Society of Automotive Engineers (SAE), "Electric Vehicle and Plug-in Hybrid Electric Vehicle Conductive Charge Coupler," J1772_201202, SAE International Standards, February 2012.

Tuffner, F.K. and M. Kintner-Meyer, "Using Electric Vehicles to Meet Balancing Requirements Associated with Wind Power," PNNL-20501, Pacific Northwest National Laboratory, Richland, WA, June 2011.

U.S. Department of Transportation (USDOT), Bureau of Transportation Statistics, NHTS 2001 Highlights Reports, BTS03-05, Washington, D.C., 2003. 
Name

Organization

Address

City, State, ZIP Code 


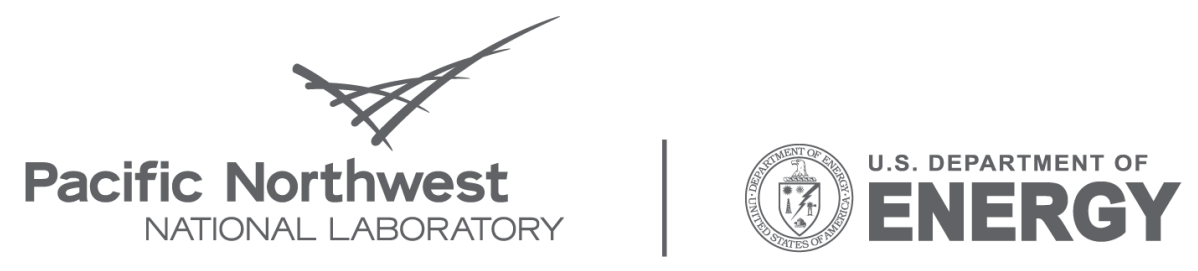

Proudly Operated by Battelle Since 1965

902 Battelle Boulevard

P.O. Box 999

Richland, WA 99352

1-888-375-PNNL (7665)

www.pnl.gov 